\title{
A New Sample of (Wandering) Massive Black Holes in Dwarf Galaxies from High-resolution Radio Observations
}

\author{
Amy E. Reines ${ }^{1}$ (D), James J. Condon ${ }^{2}$ (1) Jeremy Darling $^{3}$ (D), and Jenny E. Greene ${ }^{4}$ \\ 1 eXtreme Gravity Institute, Department of Physics, Montana State University, Bozeman, MT 59717, USA; amy.reines@montana.edu \\ ${ }^{2}$ National Radio Astronomy Observatory, Charlottesville, VA 22903, USA \\ ${ }^{3}$ Center for Astrophysics and Space Astronomy, Department of Astrophysical and Planetary Sciences, University of Colorado, 389 UCB, Boulder, \\ CO 80309-0389, USA \\ ${ }^{4}$ Department of Astrophysical Sciences, Princeton University, Princeton, NJ 08544, USA \\ Received 2019 July 9; revised 2019 August 27; accepted 2019 September 3; published 2020 January 3
}

\begin{abstract}
We present a sample of nearby dwarf galaxies with radio-selected accreting massive black holes (BHs), the majority of which are non-nuclear. We observed 111 galaxies using sensitive, high-resolution observations from the Karl G. Jansky Very Large Array (VLA) in its most extended A-configuration at $X$ band $(\sim 8-12 \mathrm{GHz})$, yielding a typical angular resolution of $\sim 0$ !' 25 and $\mathrm{rms}$ noise of $\sim 15 \mu \mathrm{Jy}$. Our targets were selected by crossmatching galaxies with stellar masses $M_{\star} \leqslant 3 \times 10^{9} M_{\odot}$ and redshifts $z<0.055$ in the NASA-Sloan Atlas with the VLA Faint Images of the Radio Sky at Twenty centimeters Survey. With our new high-resolution VLA observations, we detect compact radio sources toward 39 galaxies and carefully evaluate possible origins for the radio emission, including thermal $\mathrm{H}$ II regions, supernova remnants, younger radio supernovae, background interlopers, and active galactic nuclei (AGNs) in the target galaxies. We find that 13 dwarf galaxies almost certainly host active massive BHs, despite the fact that only one object was previously identified as having optical signatures of an AGN. We also identify a candidate dual radio AGN in a more massive galaxy system. The majority of the radio-detected BHs are offset from the center of the host galaxies, with some systems showing signs of interactions/mergers. Our results indicate that massive BHs need not always live in the nuclei of dwarf galaxies, confirming predictions from simulations. Moreover, searches attempting to constrain $\mathrm{BH}$ seed formation using observations of dwarf galaxies need to account for such a population of "wandering" BHs.
\end{abstract}

Unified Astronomy Thesaurus concepts: Intermediate-mass black holes (816); Dwarf galaxies (416); Radio sources (1358); Very Large Array (1766); Active galactic nuclei (16); AGN host galaxies (2017); Dwarf irregular galaxies (417); Supermassive black holes (1663)

Supporting material: machine-readable tables

\section{Introduction}

Massive black holes $(\mathrm{BHs})^{5}$ typically reside in the centers of massive galaxies, yet the secrets of $\mathrm{BH}$ formation have been erased in these systems by merger-driven growth over cosmic history (e.g., Volonteri 2010; Natarajan 2014). Moreover, the first "seed" BHs at high redshift (e.g., Latif \& Ferrara 2016), which can ultimately grow to millions or billions of solar masses, are too small and faint to detect with existing telescopes (e.g., Volonteri \& Reines 2016; Schleicher 2018; Vito et al. 2018). A promising alternative approach to learn about $\mathrm{BH}$ origins is to find and study the least-massive BHs in nearby dwarf galaxies, which places the most definitive constraints on the masses of $\mathrm{BH}$ seeds (see Reines \& Comastri 2016 for a review).

There are observational and theoretical arguments for why this strategy is appealing. First, we know from a number of observational scaling relations that the smallest BHs should live in low-mass galaxies (e.g., Kormendy \& Ho 2013; McConnell \& Ma 2013; Reines \& Volonteri 2015; Bentz \& Manne-Nicholas 2018; Schutte et al. 2019). Second, models of $\mathrm{BH}$ growth in a cosmological context indicate that the signatures of BH seeding should be strongest in dwarf galaxies, with the $\mathrm{BH}$ occupation fraction and scaling relations at low

\footnotetext{
5 Here, the term "massive BH" refers to a nonstellar BH. Massive BHs have so far been detected in the range of $M_{\mathrm{BH}} \sim 10^{4}-10^{10} M_{\odot}$.
}

masses varying depending on whether the seeds are light or heavy (Volonteri et al. 2008; Volonteri \& Natarajan 2009; van Wassenhove et al. 2010; Ricarte \& Natarajan 2018). Recent simulations also suggest that dwarfs should contain relatively pristine $\mathrm{BHs}$, due to supernova feedback stunting $\mathrm{BH}$ growth (Anglés-Alcázar et al. 2017; Habouzit et al. 2017). Finally, from a practical standpoint, finding and studying active massive BHs with $M_{\mathrm{BH}} \lesssim 10^{5} M_{\odot}$ in present-day dwarf galaxies is actually achievable with current capabilities (e.g., Reines et al. 2013; Baldassare et al. 2015).

In general, there are many ways to identify the presence of an accreting massive $\mathrm{BH}$ (see $\mathrm{Ho} 2008$ for a review). Broad $\mathrm{H} \alpha$ emission has been used to identify low-mass active galactic nuclei (AGNs; Greene \& Ho 2004, 2007; Dong et al. 2012; Reines et al. 2013) and accompanying narrow-line AGN signatures can be use to firmly rule out supernova interlopers (Baldassare et al. 2016). Optical narrow emission-line diagnostic diagrams (e.g., the BPT diagram after Baldwin et al. 1981) are used to separate line-emitting galaxies by their primary excitation source (AGN versus massive stars) and have been applied to the general galaxy population in the Sloan Digital Sky Survey (SDSS; e.g., Kauffmann et al. 2003; Kewley et al. 2006) and with a focus on low-mass (Barth et al. 2008) and dwarf galaxies (Reines et al. 2013; Moran et al. 2014; Sartori et al. 2015). However, optical diagnostics are only sensitive to BHs radiating at significant fractions of their Eddington luminosity, which are rare compared to lower 
Eddington-ratio systems (Schulze \& Wisotzki 2010). Furthermore, low-mass galaxies generally have ongoing star formation, gas, and dust that can mask or extinguish the optical signatures of $\mathrm{BH}$ accretion. Therefore, while there may be an accreting $\mathrm{BH}$ present at the center of a dwarf galaxy, the total observed line emission in the SDSS $3^{\prime \prime}$ aperture may be dominated by star formation. Even without significant ongoing star formation, AGN signatures may be heavily diluted by host galaxy light, such that the emission lines are effectively hidden (Moran et al. 2002). Therefore, alternative search techniques (e.g., optical variability; see Baldassare et al. 2018) and observations at other wavelengths are needed to make further progress on the demographics of massive BHs in dwarf galaxies.

X-ray observations can also be used to search for massive BHs in dwarf galaxies (Gallo et al. 2008; Schramm et al. 2013; Reines et al. 2014, 2016; Lemons et al. 2015; Miller et al. 2015; Secrest et al. 2015; Pardo et al. 2016; Baldassare et al. 2017; Chen et al. 2017; Mezcua et al. 2018; Latimer et al. 2019); however, long exposure times (hours) are typically necessary to detect lowluminosity AGN, even at modest distances. Therefore, it is currently not practical to conduct a large-scale X-ray survey of dwarf galaxies to search for low-mass BHs with correspondingly low luminosities. Moreover, X-ray observations alone cannot always firmly establish the presence of a massive $\mathrm{BH}$, because stellar-mass X-ray binaries can have luminosities comparable to weakly accreting massive BHs.

Mid-infrared searches, based on Wide-field Infrared Survey Explorer (WISE) colors for example (Satyapal et al. 2014; Sartori et al. 2015; Marleau et al. 2017; Kaviraj et al. 2019), are fraught with other problems. In principle very red mid-IR colors can be used to infer the presence of an AGN. However, at the low luminosities associated with dwarf galaxies, the interpretation is less clear, as red mid-IR colors can also be attributed to intense star formation (Hainline et al. 2016; Satyapal et al. 2018). Moreover, the resolution $\left(6^{\prime \prime}-12^{\prime \prime}\right.$ for WISE) is not sufficient to disentangle potential AGN emission from star formation-related emission. The detection of infrared coronal lines with the James Webb Space Telescope may prove to be a useful diagnostic for probing low-mass BHs (Cann et al. 2018); however, large surveys of dwarf galaxies intended to identify new low-mass AGNs will likely be prohibitively expensive.

Here, we undertake a large-scale radio search for massive BHs in dwarf galaxies using high-resolution observations from the NSF's Karl G. Jansky Very Large Array (VLA). This work is motivated in part by the discovery of the first radio (and $\mathrm{X}$-ray) detected massive $\mathrm{BH}$ in a dwarf starburst galaxy, Henize 2-10 (Reines et al. 2011; Reines \& Deller 2012; Reines et al. 2016). Furthermore, radio observations with the VLA are ideally suited for a large-scale survey of dwarf galaxies. First, AGNs (even weak ones) almost always produce radiocontinuum emission at centimeter wavelengths (Ho 2008 and references therein), which is virtually immune to dust extinction. Second, the subarcsecond spatial resolution afforded by the VLA in the A-configuration is sufficient to pinpoint the location of any compact radio emission and isolate it from the diffuse synchrotron emission emitted by the host galaxy. Finally, the exquisite sensitivity of the VLA now enables efficient observations of a large number of dwarf galaxies; this was not possible before the enormous increase in bandwidth provided by the Expanded VLA project.

\section{Sample of Dwarf Galaxies}

As in Reines et al. (2013), we construct our parent sample of dwarf galaxies from the NASA-Sloan Atlas (NSA; version v0_1_2), which is a catalog of images and parameters of SDSS galaxies with redshifts $z<0.055(D \lesssim 225 \mathrm{Mpc})$. Redshifts primarily come from SDSS DR8 spectra. However, in some cases, values are taken from other sources, ${ }^{6}$ including the NASA Extragalactic Database, the Six-degree Field Galaxy Redshift Survey, the Two-degree Field Galaxy Redshift Survey, the CfA Redshift Survey (ZCAT), and ALFALFA. Stellar masses are provided in the NSA and are derived from the kcorrect code of Blanton \& Roweis (2007), which fits broadband Galaxy Evolution Explorer (GALEX) and SDSS fluxes using templates based on the stellar population synthesis models of Bruzual \& Charlot (2003) and the nebular emissionline models of Kewley et al. (2001).

We first select sources with stellar masses $M_{\star} \leqslant 3 \times 10^{9} M_{\odot}$ in the NSA, as well as impose modest absolute magnitude cuts of $M_{g}$ and $M_{r}>-20$ to help mitigate selecting luminous/massive galaxies with erroneous mass estimates. Following Reines et al. (2013), our upper mass limit is approximately equal to the stellar mass of the Large Magellanic Cloud (LMC), which is the most massive dwarf satellite of the Milky Way. The minimum stellar mass of our sample is $M_{\star} \gtrsim 10^{7} M_{\odot}$ and is due to the SDSS spectroscopic apparent magnitude limit of $r<17.7$. Our parent sample includes 43,707 objects.

To maximize the number of detections and to target galaxies with radio luminosities similar to Henize 2-10, the prototypical dwarf galaxy with a radio-selected massive $\mathrm{BH}$ (Reines et al. 2011; Reines \& Deller 2012), we select a subsample of dwarf galaxies detected in the VLA Faint Images of the Radio Sky at Twenty-centimeters (FIRST) Survey (Becker et al. 1995). We cross-correlate our parent sample of 43,707 dwarfs to the FIRST catalog (version 2013 Jun 5), requiring a match radius $\leqslant 5^{\prime \prime}$ ( $\sim$ the resolution of FIRST), and find 186 matches throughout the NSA volume. We then visually inspect all of the matches and eliminate 35 interlopers from the sample that are clearly not dwarf galaxies (e.g., nearby H II regions and distant quasars), along with three nearby, well-studied dwarf galaxies with archival VLA data (including NGC 4395 that is already known to host an AGN), leaving us with 148 objects. Of these, 111 dwarf galaxies with FIRST detections were observed with the VLA for this program (see Figure 1 and Table 1). The remaining galaxies were not observed, due to scheduling priorities.

Our radio-selected dwarf galaxies are quite rare-only $\sim 0.3 \%$ of sources in our parent sample of dwarf galaxies have counterparts in FIRST. However, we note that many AGNs, even in low-redshift galaxies, do not produce radio continuum emission that is detectable at FIRST sensitivity levels (Condon et al. 2019), and therefore we could be missing a significant population of massive BHs. While our sample of dwarf galaxies is clearly biased, targeting a radio-selected sample virtually ensures there is either an AGN, intense star formation, or both. Even if the majority of the radio emission detected in FIRST is due to star formation, the sensitivity and high angular resolution of our VLA observations will enable the detection of massive BHs in our target dwarf galaxies, so long as they are present and accreting at modest rates.

\footnotetext{
6 http://www.nsatlas.org/documentation
} 

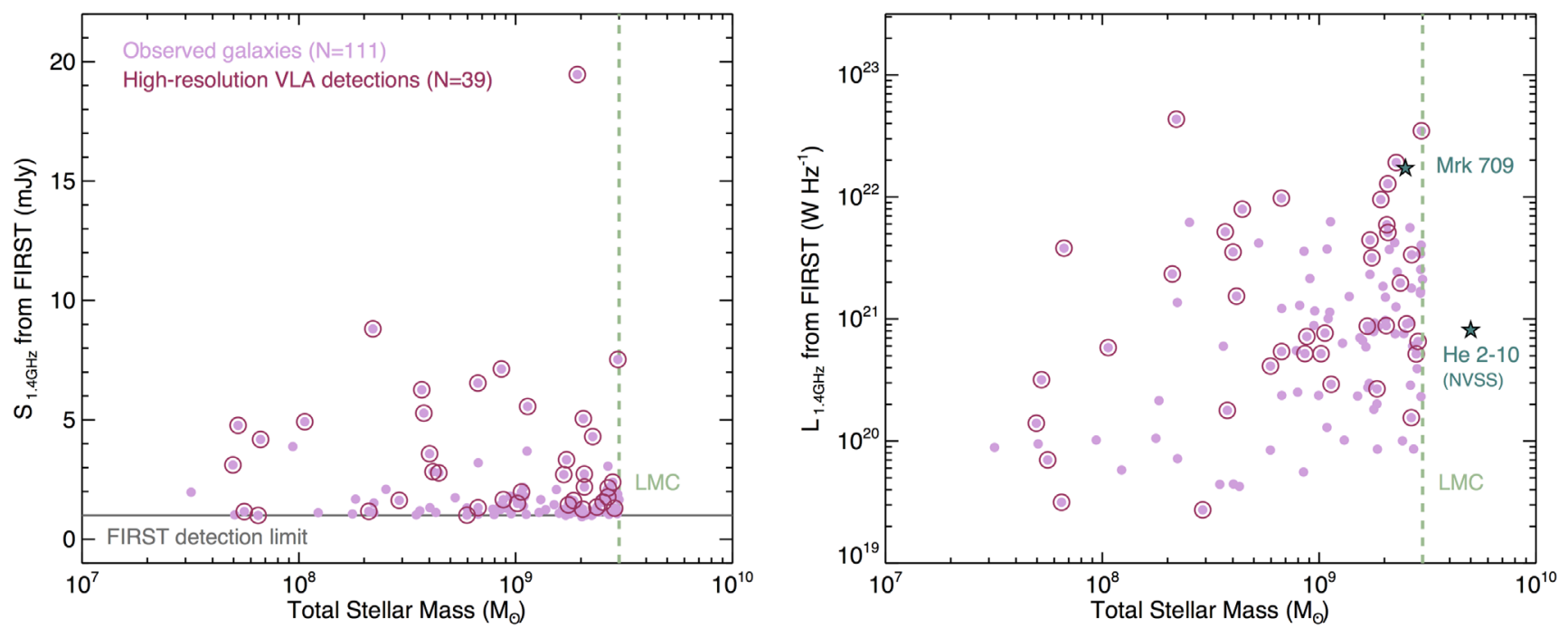

Figure 1. Peak flux density (left) and corresponding luminosity density (right) at $1.4 \mathrm{GHz}$ from the FIRST survey vs. stellar mass from the NSA for our sample of target galaxies. We detect compact radio sources with our new VLA observations at $9 \mathrm{GHz}$ toward 39 of the 111 observed galaxies (circled points). The stellar mass of the LMC is indicated as a dashed line. Henize 2-10 (Reines et al. 2011) and Mrk 709 (Reines et al. 2014) are shown as teal stars in the right panel. Henize 2-10 is not in the FIRST footprint, so we show the $1.4 \mathrm{GHz}$ NVSS flux density. Although the NVSS beam is significantly larger than FIRST, the distance to Henize 2-10 is only $\sim 9 \mathrm{Mpc}$ and the median distance to our VLA sample is $\sim 70 \mathrm{Mpc}$. Therefore, the physical resolution is similar.

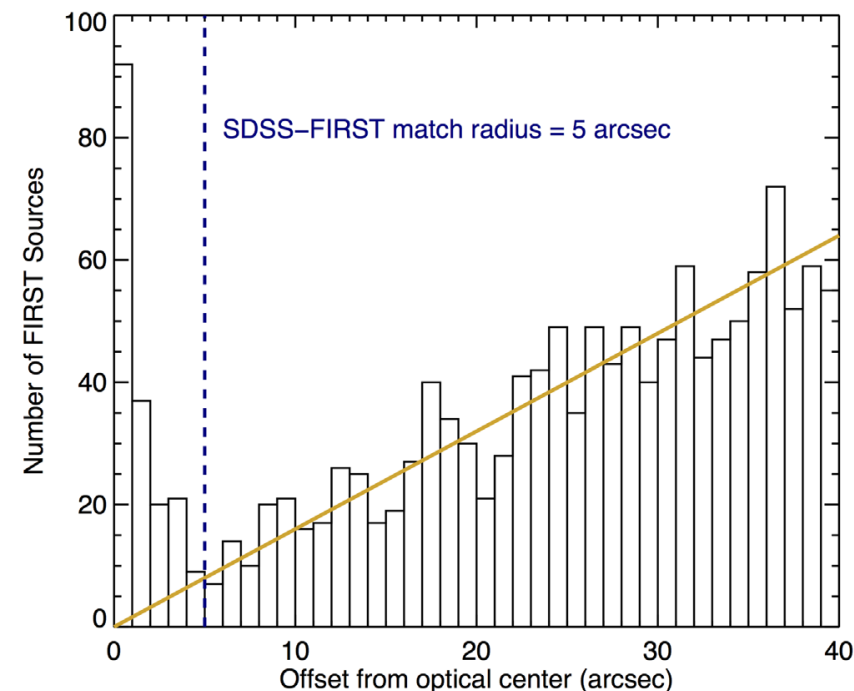

Figure 2. Observed offset distribution from cross-matching our SDSS parent sample with the FIRST survey, using a match radius of $40^{\prime \prime}$. We select our target galaxies to have offsets $\leqslant 5^{\prime \prime}$ (blue line). The yellow line shows the expected number of chance alignments with background sources as a function of offset between an SDSS and FIRST source.

\subsection{Potential Contamination from Background Radio Sources}

We use two methods to assess the likelihood of chance alignments between galaxies in our parent sample and background radio sources. First, we estimate $N\left(S_{\min }\right)$, the cumulative number of radio source counts per steradian with $1.4 \mathrm{GHz}$ flux densities greater than $S_{\min }$, using the results of Condon (1984). Taking $S_{\min }=1.5 \mathrm{mJy}$ (i.e., the completeness limit of FIRST) and multiplying $N\left(S_{\min }\right) \approx 219,000 \mathrm{str}^{-1}$ by the area enclosed by a $5^{\prime \prime}$ circle gives the expected number of background sources for a given galaxy, $N_{\mathrm{bk}, \text { gal }}=0.0004$. Across our entire parent sample of 43,707 galaxies, we expect $N_{\text {bk,samp }} \approx 18 \pm \sqrt{18} \approx 18 \pm 4$ background sources.

We also estimate the expected number of background sources using the data in Figure 2, which shows the observed offset distribution from cross-matching our SDSS sample and FIRST using a match radius of $40^{\prime \prime}$. The offset probability histogram for background sources should be a Rayleigh distribution, which equals zero at an offset of zero and rises linearly for small offsets (see the yellow line in Figure 2). The observed distribution is a minimum at our adopted match radius of $5^{\prime \prime}$. At larger offsets, the number of sources per arcsecond of offset, $N\left(d_{\text {off }}\right)$, is well-fit by $N\left(d_{\text {off }}\right)=1.6 d_{\text {off }}$, where the offset $d_{\text {off }}$ is in units of arcsec. The total estimated number of background sources with offsets less than $5^{\prime \prime}$ is found by integrating $N\left(d_{\text {off }}\right)$ from $d_{\text {off }}=0^{\prime \prime}$ to $5^{\prime \prime}$, which gives $N_{\text {bk,samp }} \approx 20 \pm \sqrt{20} \approx 20 \pm 4$ sources. This is consistent with our calculation above using known radio source counts, and we adopt the average of $N_{\mathrm{bk}, \text { samp }} \approx 19 \pm 4$.

The $19 \pm 4$ chance alignments with background radio sources are expected to be present in the 186 initial matches between our parent sample of 43,707 SDSS objects and FIRST radio sources (using a match radius of $5^{\prime \prime}$ ). Given that we obtained new VLA observations for only 111 galaxies of the 186 matches, we expect $111 / 186 \times 19 \approx 11 \pm 3$ background sources to be present in our observed sample (i.e., roughly $10 \%$ contamination). The background contaminants will be dominated by AGNs/quasars at FIRST flux densities $S_{1.4 \mathrm{GHz}} \gtrsim$ 1 mJy; e.g., see Figure 2 in Becker et al. (1995).

It is not immediately clear whether or not we would detect these background AGNs with our new high-resolution VLA observations at $9 \mathrm{GHz}$. If the FIRST flux density is dominated by a compact flat spectrum core, then we expect we would. However, if the FIRST flux density at $5^{\prime \prime}$ scales is dominated by more extended steep spectrum lobes, we may not detect the source at high $(\sim 0$ !' 25$)$ resolution. Therefore, we consider the $11 \pm 3$ expected background sources in our observed sample to be an upper limit on the number of background sources in our detected sample. As discussed in Section 4, we detect $9 \mathrm{GHz}$ compact radio emission toward 39 of the 111 observed galaxies. We find two confirmed background sources and two additional strong background candidates (see Figure 3 and footnotes 6-9), leaving no more than $\sim 7$ contaminants left in the remaining 35 galaxies. 
Table 1

Observed Dwarf Galaxy Sample with FIRST Detections

\begin{tabular}{|c|c|c|c|c|c|c|c|c|c|c|c|c|}
\hline \multirow[b]{2}{*}{$\begin{array}{l}\text { ID } \\
\text { (1) }\end{array}$} & \multirow[b]{2}{*}{$\begin{array}{c}\text { Name } \\
(2)\end{array}$} & \multicolumn{9}{|c|}{ NSA (optical) } & \multicolumn{2}{|c|}{ FIRST (radio) } \\
\hline & & $\begin{array}{c}\text { NSAID } \\
\text { (3) }\end{array}$ & $\begin{array}{l}\text { R.A. } \\
(4)\end{array}$ & $\begin{array}{c}\text { Decl. } \\
(5)\end{array}$ & $\begin{array}{c}z \\
(6)\end{array}$ & $\begin{array}{c}\log M_{\star} \\
\quad(7)\end{array}$ & $\begin{array}{l}M_{g} \\
(8)\end{array}$ & $\begin{array}{c}g-r \\
(9)\end{array}$ & $\begin{array}{c}r_{50} \\
(10)\end{array}$ & $\begin{array}{c}\text { Sérsic } n \\
(11)\end{array}$ & $\begin{array}{l}S_{1.4 \mathrm{GHz}} \\
\quad(12)\end{array}$ & $\begin{array}{c}\text { Offset } \\
(13)\end{array}$ \\
\hline 1 & J0018-0903 & 21913 & 4.53817 & -9.05993 & 0.0233 & 9.05 & -18.42 & 0.45 & 0.77 & 5.9 & 0.71 & 0.7 \\
\hline 2 & $\mathrm{~J} 0019+1507$ & 26027 & 4.75134 & 15.11978 & 0.0376 & 8.65 & -18.42 & 0.15 & 1.51 & 0.8 & 2.58 & 4.3 \\
\hline 3 & J0032-0656 & 127158 & 8.20313 & -6.94158 & 0.0162 & 9.42 & -18.65 & 0.39 & 1.09 & 1.0 & 2.49 & 2.4 \\
\hline 4 & $\mathrm{~J} 0048+0037$ & 62105 & 12.12363 & 0.61966 & 0.0383 & 8.40 & -17.67 & -0.03 & 0.95 & 0.5 & 2.86 & 3.9 \\
\hline 5 & J0049-0242 & 127796 & 12.46603 & -2.71196 & 0.0131 & 9.01 & -16.75 & 0.68 & 0.47 & 5.0 & 0.96 & 1.3 \\
\hline 6 & $\mathrm{~J} 0106+0046$ & 23750 & 16.52989 & 0.77606 & 0.0171 & 9.40 & -18.42 & 0.39 & 5.01 & 1.8 & 1.47 & 2.2 \\
\hline 7 & J0111-0139 & 128822 & 17.95825 & -1.65511 & 0.0158 & 9.46 & -19.12 & 0.52 & 1.29 & 3.7 & 0.73 & 0.9 \\
\hline 8 & J0115-1002 & 22511 & 18.75235 & -10.03804 & 0.0232 & 8.98 & -19.08 & 0.14 & 1.45 & 1.0 & 1.58 & 1.3 \\
\hline 9 & $\mathrm{~J} 0118+1212$ & 129053 & 19.50442 & 12.20686 & 0.0193 & 9.35 & -19.13 & 0.13 & 0.66 & 3.6 & 1.03 & 1.2 \\
\hline 10 & J0134-0741 & 130010 & 23.53643 & -7.69597 & 0.0156 & 9.28 & -16.87 & 1.07 & 0.94 & 6.0 & 20.62 & 0.1 \\
\hline
\end{tabular}

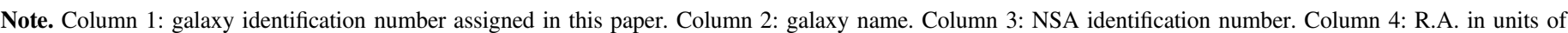

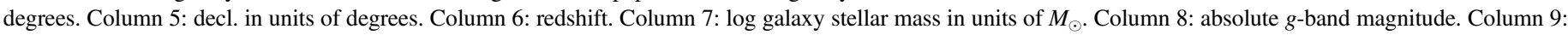

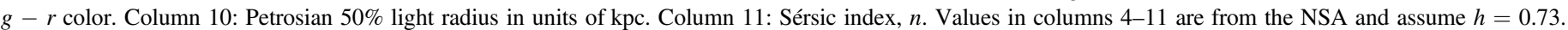

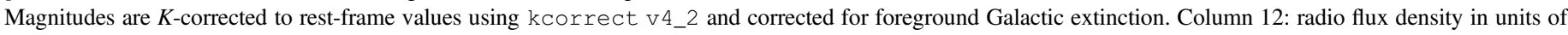
mJy at $1.4 \mathrm{GHz}$ from the FIRST survey. Column 13: offset from optical position of galaxy in units of arcseconds.

(This table is available in its entirety in machine-readable form.)

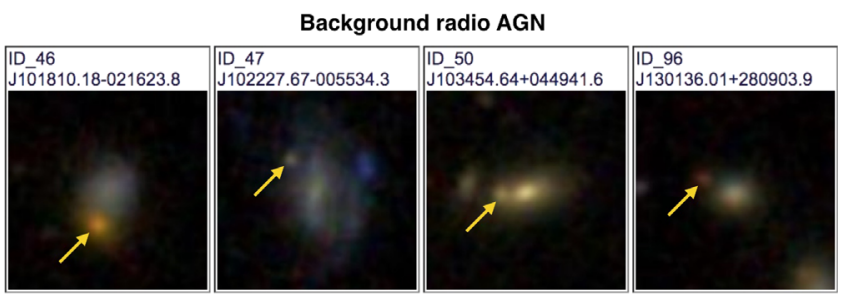

Figure 3. Identifiable background radio contaminants are found in 4 of our 39 galaxies with $9 \mathrm{GHz}$ VLA detections. The yellow arrows indicate the position of the compact radio emission. The radio sources have clear optical counterparts and are offset by 2 ". $8-4$ !" 9 from the optical centers of the target galaxies. Images are $25^{\prime \prime} \times 25^{\prime \prime}$. See the Appendix for a discussion of ID 47, which is a dwarf galaxy hosting an optical AGN.

\section{Observations and Data Reduction}

Our 111 target dwarf galaxies were observed with the VLA in the most extended A-configuration in 2014 from February 20 to May 31 (PI: Reines; Project 14A-220). Continuum observations were carried out at $X$ band with $2 \times 2 \mathrm{GHz}$ basebands centered at 9.0 and $10.65 \mathrm{GHz}$. These observing parameters yield a resolution of $\sim 0$ ". 25 , and therefore the beam solid angle is $\sim 400 \times$ smaller than FIRST. At the median distance of our sample of dwarf galaxies $(\sim 70 \mathrm{Mpc})$, this angular resolution corresponds to a physical scale of $\sim 85 \mathrm{pc}$.

We grouped the observations into $1 \mathrm{hr}$-long scheduling blocks to facilitate dynamic scheduling, typically with five target galaxies per group. On-source integration times were $\sim 6-7$ minutes per galaxy yielding a typical rms noise of $\sim 15 \mu \mathrm{Jy}$ beam $^{-1}(\sim 10 \times$ more sensitive than FIRST). Nearby phase calibrators were observed for $\sim 1.5$ minutes before and after each target. A flux calibrator was also observed during each block. The absolute flux calibration of the VLA data is expected to be $\sim 5 \%$.

The raw data were reduced using CASA version 4.7.2 on the NRAO New Mexico Array Science Center computer cluster. We first ran the VLA calibration pipeline on each of our 23 scheduling blocks. Additional flagging was then performed upon inspection of the data and the pipeline was rerun with the additional flags preapplied. The final calibrated science target data were split off into the two basebands and imaged separately using multifrequency synthesis and Briggs weighting. A summary of our VLA observations is given in Table 2.

\section{Compact Radio Sources}

We detect a total of 48 compact radio sources toward 39 of our 111 target galaxies (i.e., a 35\% detection rate), with a detection threshold of $\gtrsim 3 \sigma$. We use the term "compact" radio sources to differentiate between our VLA detections at high resolution and the radio sources detected toward our target galaxies at lower resolution in the FIRST survey. The VLA images are shown in Figure 4. We identify four radio detections that appear to be unrelated background objects with point-like optical counterparts (IDs 46, $47,,^{8} 50,{ }^{9} 96^{10}$; see Figure 3 and the Appendix). For the remaining 35 galaxies with VLA detections, we visually inspect optical or H I spectra (see Section 2) to verify redshifts - and hence, stellar masses. We are able to confirm that 28 have reliable redshifts and are bona fide dwarf galaxies. The remaining seven galaxies have unreliable redshifts due to poor quality spectra and/or photometric redshifts with relatively large uncertainties. We refer to the restricted sample of 28 confirmed dwarf galaxies (with 35 compact radio sources) as Sample A, and the remaining seven galaxies (with nine compact radio sources) as Sample B (see Table 3).

Radio flux densities at 9.0 and $10.65 \mathrm{GHz}$ are measured interactively in the CASA viewer, using two different methods. First, we attempt to fit the detected sources with a single Gaussian model, using the task IMFIT. This provides a good fit

\footnotetext{
7 We confirm a background radio source toward ID 46. An SDSS/BOSS spectrum of the offset source indicated by the arrow in Figure 3 gives a redshift of $z=0.366$.

8 We confirm that the offset radio source toward ID 47 is a background AGN. See the Appendix.

9 An SDSS spectrum of the target galaxy ID 50 centered in Figure 3 indicates a redshift of $z=0.102$, and therefore it is unlikely to be a dwarf galaxy. We do not know the redshift of the offset source.

${ }^{10}$ The redshift of the target galaxy ID 96 and the offset source are unknown. Given the large offset and clear optical counterpart of the radio source, the radio source is likely unrelated to the target galaxy.
} 


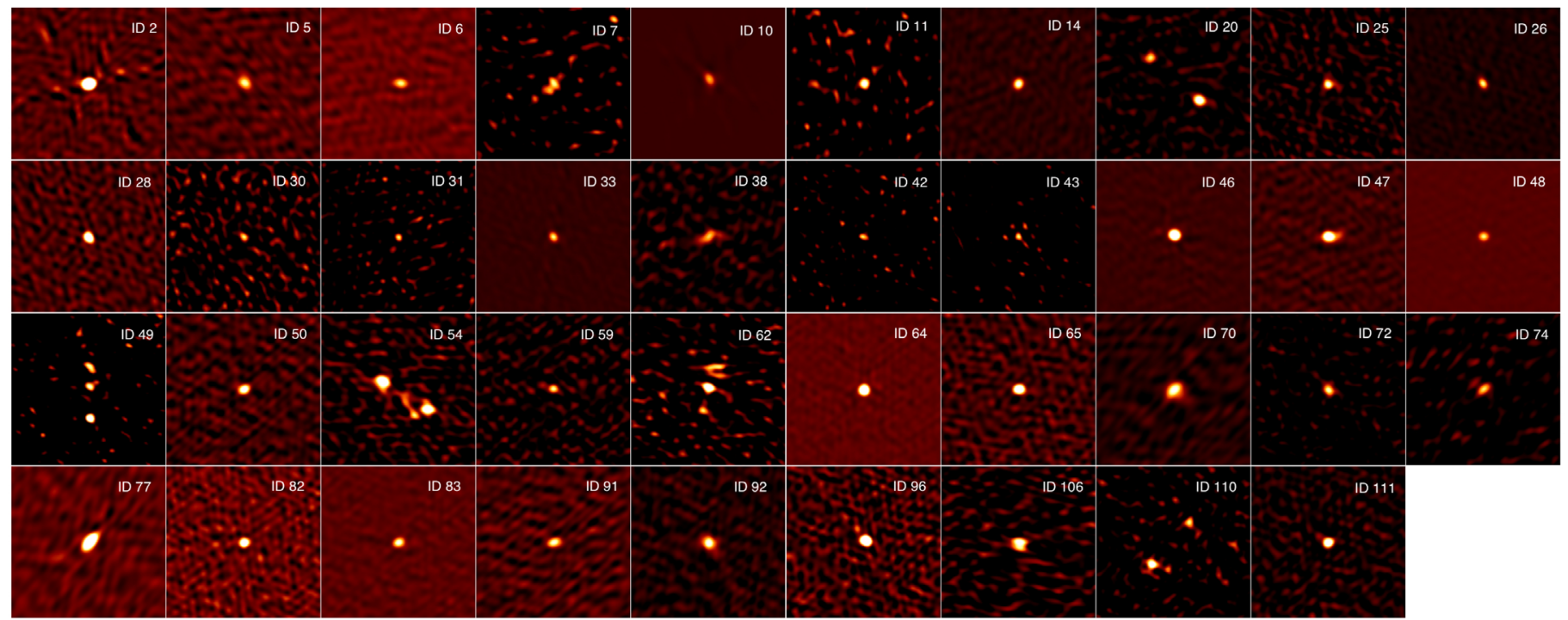

Figure 4. Panels show $9 \mathrm{GHz}$ VLA images of the compact radio sources detected toward our target galaxies, centered on the radio emission. These images include the objects in Sample A and Sample B, as well as the likely background contaminants toward the galaxies in Figure 3. All sources are within 5" of the optical center of the galaxy. Images are $4^{\prime \prime} \times 4^{\prime \prime}$, and the typical synthesized beam size is $\sim 0$.' 25 . See Table 3 for source properties.

Table 2

VLA Observations

\begin{tabular}{|c|c|c|c|c|c|c|c|c|c|c|}
\hline \multirow[b]{2}{*}{ ID } & \multirow[b]{2}{*}{ Name } & \multirow[b]{2}{*}{$\begin{array}{c}\text { Date } \\
\text { Observed }\end{array}$} & \multirow[b]{2}{*}{$\begin{array}{c}\text { Flux } \\
\text { Calibrator }\end{array}$} & \multirow[b]{2}{*}{$\begin{array}{l}\text { Phase } \\
\text { Calibrator }\end{array}$} & \multicolumn{3}{|c|}{$9 \mathrm{GHz}$} & \multicolumn{3}{|c|}{$10.65 \mathrm{GHz}$} \\
\hline & & & & & $\begin{array}{c}\text { Synthesized } \\
\text { Beam }\end{array}$ & P.A. & $\begin{array}{c}\text { rms } \\
\text { Noise }\end{array}$ & $\begin{array}{c}\text { Synthesized } \\
\text { Beam }\end{array}$ & P.A. & $\begin{array}{c}\text { rms } \\
\text { Noise }\end{array}$ \\
\hline 3 & J0032-0656 & 2014 Feb 21 & $3 \mathrm{C} 48$ & J0006-0623 & $0.26 \times 0.18$ & 41 & 16 & $0.22 \times 0.15$ & 44 & 17 \\
\hline 4 & $\mathrm{~J} 0048+0037$ & 2014 Mar 9 & $3 \mathrm{C} 48$ & J0115-0127 & $0.24 \times 0.18$ & 65 & 24 & $0.21 \times 0.16$ & 69 & 28 \\
\hline 5 & J0049-0242 & 2014 Mar 9 & $3 \mathrm{C} 48$ & $\mathrm{~J} 0115-0127$ & $0.26 \times 0.18$ & 59 & 15 & $0.22 \times 0.16$ & 59 & 29 \\
\hline 8 & J0115-1002 & 2014 Feb 21 & $3 \mathrm{C} 48$ & J0116-1136 & $0.25 \times 0.17$ & 27 & 16 & $0.21 \times 0.15$ & 28 & 17 \\
\hline 9 & $\mathrm{~J} 0118+1212$ & 2014 Feb 21 & $3 \mathrm{C} 48$ & $\mathrm{~J} 0121+0422$ & $0.19 \times 0.17$ & -86 & 15 & $0.16 \times 0.15$ & -83 & 16 \\
\hline 10 & J0134-0741 & 2014 Mar 9 & $3 \mathrm{C} 48$ & J0116-1136 & $0.27 \times 0.18$ & 30 & 20 & $0.23 \times 0.16$ & 31 & 20 \\
\hline
\end{tabular}

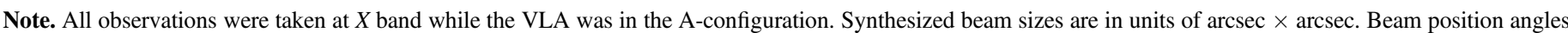
are in units of degrees. Here, rms noise has units of $\mu \mathrm{Jy}_{\text {beam }}{ }^{-1}$.

(This table is available in its entirety in machine-readable form.)

for the majority of sources. At the resolution of our observations $(\sim 0$ !' 25$)$, many of the detections are consistent with being point sources (Table 3 ) as determined from IMFIT. We also measure flux densities with a variety of user-defined apertures that trace the shape of the source. This is particularly useful for those sources with extended or irregularly shaped emission profiles.

Table 3 lists properties of the detected compact radio sources, including coordinates, flux densities, luminosity densities, spectral indices, and radio-optical positional offsets. The $9 \mathrm{GHz}$ spectral luminosities in Sample $\mathrm{A}$ are in the range $L_{9 \mathrm{GHz}} \sim 10^{18.5}-10^{22.0} \mathrm{~W} \mathrm{~Hz}^{-1}$ with a median value of $10^{19.6} \mathrm{~W} \mathrm{~Hz}^{-1}$ (see Figure 5). For comparison, the lowluminosity AGN in Henize 2-10 has a spectral luminosity of $\sim 10^{19} \mathrm{~W} \mathrm{~Hz}^{-1}$. We calculate spectral indices, $\alpha$, between 9.0 and $10.65 \mathrm{GHz}$ using the measured flux densities in each baseband $\left(S_{\nu} \propto \nu^{\alpha}\right)$. Given the small spread in frequency and typical signal-to-noise ratios, the uncertainties are rather large, except for the most luminous sources (Table 3). We detect multiple compact radio sources in three galaxies in Sample A and two additional galaxies in Sample B.

By design, all of the radio sources are within 5 " of the optical center of the galaxy, as defined by the SDSS images. The observed distribution of radio-optical positional offsets for all 44 detections (after removing identifiable background contaminants) is shown in the right panel of Figure 5. We expect at most $7 \pm 3$ remaining background radio contaminants in Samples A and B, where the probability of contamination rises linearly with separation between the radio source and the optical center of the galaxy. We construct the corresponding offset distribution of possible background contaminants by scaling the relation shown in Figure 2 such that $N\left(d_{\text {off }}\right)=$ $A \times 1.6 d_{\text {off }}$, where $A$ is the scale factor and $d_{\text {off }}$ is the offset in arcsec. Integrating between $0^{\prime \prime}$ and $5^{\prime \prime}$ and setting the result 
Table 3

Compact Radio Sources Detected toward Target Galaxies

\begin{tabular}{|c|c|c|c|c|c|c|c|c|c|}
\hline $\begin{array}{l}\text { ID } \\
\text { (1) }\end{array}$ & $\begin{array}{l}\text { Name } \\
(2)\end{array}$ & $\begin{array}{l}\text { R.A. } \\
\text { (3) }\end{array}$ & $\begin{array}{l}\text { Decl. } \\
\text { (4) }\end{array}$ & $\begin{array}{l}\text { Offset } \\
(5)\end{array}$ & $\begin{array}{l}S_{9 \mathrm{GHz}} \\
\text { (6) }\end{array}$ & $\begin{array}{c}\log L_{9 \mathrm{GHz}} \\
\text { (7) }\end{array}$ & $\begin{array}{c}\alpha \\
(8)\end{array}$ & $\begin{array}{l}\text { Point Source } \\
\text { (9) }\end{array}$ & $\begin{array}{l}\text { Note } \\
(10)\end{array}$ \\
\hline 2 & J0019+1507 & 4.74994 & 15.11973 & 4.9 & $2586(27)$ & 21.9 & $-0.2(0.1)$ & True & Sample A, AGN \\
\hline 5 & J0049-0242 & 12.46602 & -2.71195 & 0.0 & $372(34)$ & 20.1 & $-1.9(0.9)$ & False & Sample B, AGN \\
\hline 6 & $\mathrm{~J} 0106+0046$ & 16.53045 & 0.77620 & 2.1 & $352(24)$ & 20.3 & $-0.5(0.6)$ & True & Sample A, AGN \\
\hline 7 & J0111-0139 & 17.95820 & -1.65520 & 0.4 & $172(20)$ & 19.9 & $-1.2(1.1)$ & False & Sample A, SF \\
\hline 10 & J0134-0741 & 23.53642 & -7.69593 & 0.1 & $21831(200)$ & 22.0 & $0.1(0.1)$ & True & Sample B, AGN \\
\hline 11 & J0205-0750 & 31.35838 & -7.83610 & 1.0 & $126(37)$ & 19.6 & $-2.7(1.9)$ & True & Sample A, SF \\
\hline 14 & $\mathrm{~J} 0247+0535$ & 41.94719 & 5.58766 & 1.4 & $1148(31)$ & 19.7 & $-1.0(0.3)$ & True & Sample B, AGN \\
\hline $20 \mathrm{a}$ & J0854-0240 & 133.63228 & -2.68335 & 0.2 & $379(30)$ & 20.3 & $-0.8(0.9)$ & False & Sample B, AGN \\
\hline $20 \mathrm{~b}$ & $\ldots$ & 133.63264 & -2.68304 & 1.9 & 199(28) & 20.0 & $-2.0(1.5)$ & True & Sample B, AGN \\
\hline 25 & $\mathrm{~J} 0903+4824$ & 135.80403 & 48.40381 & 2.2 & $375(43)$ & 20.7 & $-1.3(1.2)$ & False & Sample A, AGN \\
\hline 26 & J0906+5610 & 136.55737 & 56.17087 & 0.4 & $929(26)$ & 21.6 & $-1.0(0.3)$ & True & Sample A, AGN \\
\hline 28 & J0909+5655 & 137.28621 & 56.92215 & 2.7 & $595(22)$ & 21.1 & $-1.1(0.4)$ & True & Sample A, AGN \\
\hline 30 & J0916+5946 & 139.18317 & 59.77447 & 1.0 & $65(21)$ & 19.4 & $\ldots$ & True & Sample A, SF \\
\hline 31 & J0926+1923 & 141.50447 & 19.38290 & 0.2 & 63(19) & 19.0 & $\ldots$ & True & Sample A, SF \\
\hline 33 & $\mathrm{~J} 0931+5633$ & 142.91008 & 56.55552 & 1.1 & $1951(27)$ & 22.0 & $-1.0(0.1)$ & True & Sample A, AGN \\
\hline 38 & J0944-0038 & 146.00783 & -0.64223 & 0.1 & $259(10)$ & 19.2 & $-1.3(0.4)$ & False & Sample A, SF \\
\hline 42 & $\mathrm{~J} 1002+4311$ & 150.70386 & 43.18802 & 1.0 & $66(23)$ & 19.7 & $\ldots$ & True & Sample A, SF \\
\hline 43 & $\mathrm{~J} 1003+5926$ & 150.96643 & 59.43628 & 0.5 & $70(30)$ & 19.2 & $-0.8(3.2)$ & True & Sample A, SF \\
\hline 46 & $\mathrm{~J} 1018-0216$ & 154.54269 & -2.27437 & 4.0 & $5444(33)$ & 22.4 & $-0.1(0.1)$ & True & Background \\
\hline 47 & $\mathrm{~J} 1022-0055$ & 155.61610 & -0.92510 & 4.9 & $1559(39)$ & 21.9 & $-1.4(0.2)$ & False & Background \\
\hline 48 & $\mathrm{~J} 1027+0112$ & 156.92241 & 1.20179 & 4.2 & $2587(33)$ & 21.4 & $-0.3(0.1)$ & True & Sample A, AGN \\
\hline $49 a$ & $\mathrm{~J} 1032+5424$ & 158.13327 & 54.40040 & 1.1 & $108(22)$ & 18.9 & $-3.1(2.0)$ & True & Sample A, SF \\
\hline $49 b$ & $\ldots$ & 158.13329 & 54.40063 & 0.3 & $102(43)$ & 18.9 & $0.0(3.8)$ & False & Sample A, SF \\
\hline $49 c$ & $\ldots$ & 158.13327 & 54.40077 & 0.2 & $100(15)$ & 18.9 & $-0.4(0.9)$ & False & Sample A, SF \\
\hline 50 & $\mathrm{~J} 1034+0449$ & 158.72845 & 4.82818 & 2.8 & $375(26)$ & 20.8 & $-1.4(0.8)$ & True & Background \\
\hline $54 \mathrm{a}$ & $\mathrm{J} 1045+5557$ & 161.34189 & 55.96118 & 0.1 & $458(64)$ & 19.2 & $-0.8(1.1)$ & False & Sample A, SF \\
\hline $54 \mathrm{~b}$ & $\ldots$ & 161.34129 & 55.96098 & 1.3 & $340(30)$ & 19.1 & $-1.7(0.6)$ & False & Sample A, SF \\
\hline $54 \mathrm{c}$ & $\ldots$ & 161.34145 & 55.96095 & 1.2 & $103(30)$ & 18.5 & $-1.6(1.9)$ & False & Sample A, SF \\
\hline $54 d$ & $\ldots$ & 161.34158 & 55.96105 & 0.7 & $99(40)$ & 18.5 & $-0.9(4.2)$ & False & Sample A, SF \\
\hline 59 & $\mathrm{~J} 1108+0026$ & 167.23329 & 0.44485 & 3.2 & $141(26)$ & 19.7 & $-1.0(1.6)$ & True & Sample A, SF \\
\hline $62 \mathrm{a}$ & J1126+5909 & 171.68445 & 59.15566 & 0.5 & $173(31)$ & 19.0 & $-1.5(1.5)$ & False & Sample A, SF \\
\hline $62 b$ & $\ldots$ & 171.68434 & 59.15581 & 1.1 & $140(26)$ & 18.9 & $-0.2(2.2)$ & False & Sample A, SF \\
\hline $62 \mathrm{c}$ & $\ldots$ & 171.68451 & 59.15549 & 0.1 & $75(28)$ & 18.6 & $-2.0(2.8)$ & True & Sample A, SF \\
\hline 64 & $\mathrm{~J} 1136+1252$ & 174.20219 & 12.87775 & 0.9 & $3218(27)$ & 21.9 & $0.1(0.1)$ & True & Sample A, AGN \\
\hline 65 & $\mathrm{~J} 1136+2643$ & 174.17740 & 26.72657 & 2.9 & $517(24)$ & 21.1 & $-1.9(0.5)$ & False & Sample A, AGN \\
\hline 70 & $\mathrm{~J} 1143-0325$ & 175.82785 & -3.43129 & 0.3 & $1009(46)$ & 19.8 & $-1.3(0.5)$ & False & Sample B, AGN \\
\hline 72 & $\mathrm{~J} 1145+3117$ & 176.40404 & 31.29970 & 0.6 & $301(41)$ & 19.4 & $-1.4(1.4)$ & False & Sample A, SF \\
\hline 74 & $\mathrm{~J} 1152-0228$ & 178.15494 & -2.46945 & 0.1 & $180(33)$ & 18.8 & $-1.2(1.7)$ & True & Sample A, SF \\
\hline 77 & J1200-0341 & 180.24292 & -3.68846 & 2.2 & $1496(33)$ & 21.3 & $-0.9(0.2)$ & True & Sample A, AGN \\
\hline 82 & $\mathrm{~J} 1220+3020$ & 185.04694 & 30.33564 & 0.1 & $397(24)$ & 20.8 & $-0.7(0.6)$ & True & Sample A, AGN \\
\hline 83 & $\mathrm{~J} 1226+0815$ & 186.51518 & 8.25528 & 0.0 & $780(26)$ & 21.0 & $-0.6(0.3)$ & True & Sample A, AGN \\
\hline 91 & $\mathrm{~J} 1253+0427$ & 193.31082 & 4.46313 & 0.5 & $312(30)$ & 18.7 & $-0.7(0.9)$ & True & Sample A, SF \\
\hline 92 & $\mathrm{~J} 1253-0312$ & 193.27487 & -3.21632 & 0.1 & $1160(76)$ & 21.1 & $-0.8(0.6)$ & False & Sample A, AGN \\
\hline 96 & $\mathrm{~J} 1301+2809$ & 195.40129 & 28.15157 & 4.3 & $783(27)$ & 20.8 & $-1.3(0.3)$ & True & Background \\
\hline 106 & $\mathrm{~J} 1718+3011$ & 259.72269 & 30.19341 & 0.1 & $381(51)$ & 20.2 & $-1.6(1.2)$ & False & Sample A, SF \\
\hline $110 \mathrm{a}$ & $\mathrm{J} 2315+0654$ & 348.93619 & 6.91072 & 1.4 & $250(10)$ & 19.5 & $-0.5(0.8)$ & False & Sample B, SF \\
\hline $110 \mathrm{~b}$ & $\ldots$ & 348.93591 & 6.91103 & 0.0 & $112(10)$ & 19.1 & $0.4(1.1)$ & False & Sample B, SF \\
\hline 111 & $\mathrm{~J} 2359+1435$ & 359.93811 & 14.58394 & 0.2 & $442(26)$ & 20.3 & $-0.7(0.6)$ & False & Sample B, AGN \\
\hline
\end{tabular}

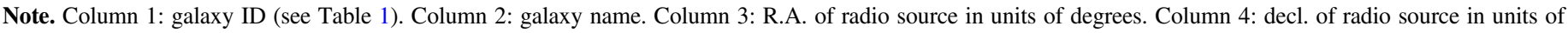

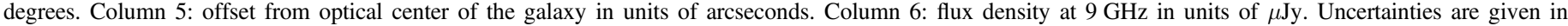

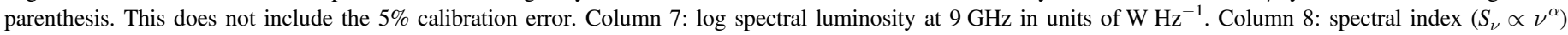

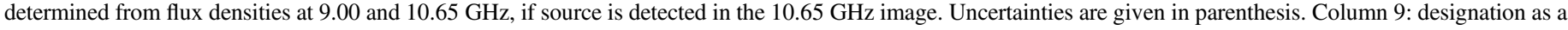

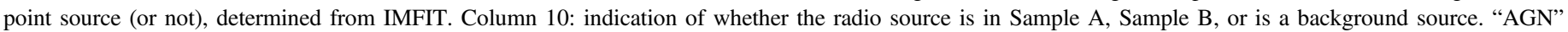
indicates radio sources that are too luminous to be consistent with star formation. "SF" indicates radio sources consistent with star formation.

equal to seven sources gives $A=0.35$. The resulting distribution of possible background sources, which we consider an upper limit, is shown in Figure 5.

We do not detect radio emission with our new highresolution VLA observations toward 72 of the 111 target galaxies with FIRST detections. In these cases, the FIRST sources are likely dominated by more extended and diffuse radio emission from star formation processes (or possibly largescale AGN radio lobes), and may include some fraction of the expected background contaminants. While we cannot rule out 

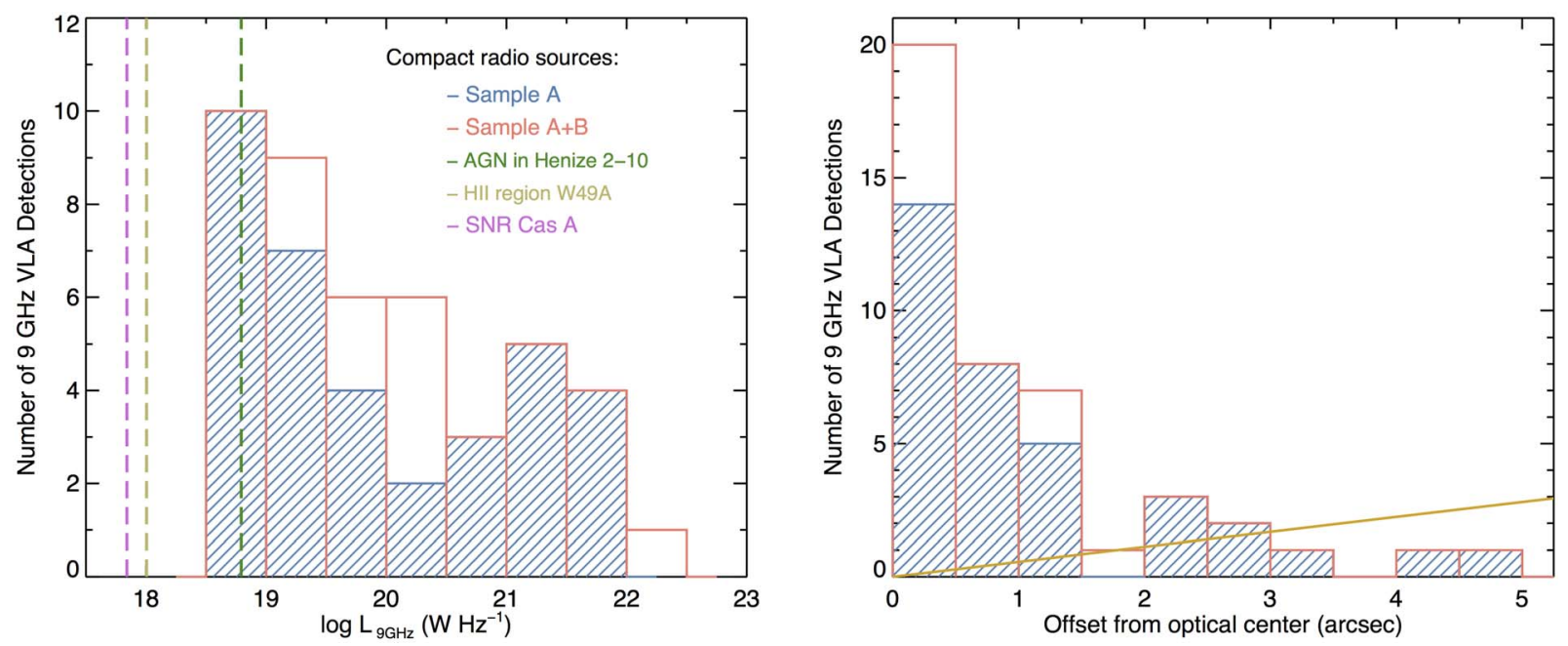

Figure 5. Left: distribution of the $9 \mathrm{GHz}$ spectral luminosities of the compact radio sources detected in our VLA observations. For comparison, we show the values of the low-luminosity AGN in Henize 2-10, the young Galactic supernova remnant Cas A, and the young Galactic $\mathrm{H}$ II region W49A. Right: distribution of the offsets between the positions of detected compact radio sources and optical positions of the target galaxies (see Table 3). The yellow line shows the expected distribution of background radio sources under the most conservative assumptions discussed in Sections 2.1 and 4.

the presence of massive $\mathrm{BHs}$ in these 72 galaxies, we have no evidence for their existence.

\section{Origin of the Compact Radio Emission}

Our primary goal is to detect compact radio emission from accreting massive $\mathrm{BHs}$ in our target galaxies. However, at centimeter radio wavelengths, we need to consider possible contamination in our sample from supernova remnants (SNRs), young supernovae $(\mathrm{SNe})$, and ionized gas from $\mathrm{H}$ II regions. Stellar-mass X-ray binaries are not a concern, as they do not exceed $\sim 10^{17} \mathrm{~W} \mathrm{~Hz}^{-1}$ in the radio even when flaring (Corbel et al. 2012; Gallo et al. 2018). Out of 44 detected compact radio sources (see Section 4), we expect at most $\sim 7 \pm 3$ background contaminants that are primarily at large $\left(\gtrsim 2^{\prime \prime}\right)$ radio-optical positional offsets (Figure 5; Sections 2.1 and 4). As demonstrated below, at least 13 compact radio sources in Sample A are almost certainly due to AGN activity. Another six (likely more massive) galaxies in Sample B also have detectable radio AGNs, with one of these systems hosting a possible dual AGN.

\subsection{Thermal H II Regions?}

We begin by considering whether the detected compact radio emission could be due to thermal bremsstrahlung (i.e., free-free emission) from ionized hydrogen in star-forming regions. Under the assumption that the detected compact radio emission is thermal bremsstrahlung from $\mathrm{H}$ II regions, we can estimate the production rate of Lyman continuum (i.e., ionizing) photons, which in turn can be used to estimate the stellar content and instantaneous star formation rates (SFRs) in these regions. The production rate of Lyman continuum photons, $Q_{\mathrm{Lyc}}$, is given by Condon (1992):

$$
\begin{aligned}
\left(\frac{Q_{\mathrm{Lyc}}}{\mathrm{s}^{-1}}\right) & \gtrsim 6.3 \times 10^{52}\left(\frac{T_{e}}{10^{4} \mathrm{~K}}\right)^{-0.45}\left(\frac{\nu}{\mathrm{GHz}}\right)^{0.1} \\
& \times\left(\frac{L_{\nu, \text { thermal }}}{10^{20} \mathrm{~W} \mathrm{~Hz}^{-1}}\right),
\end{aligned}
$$

where the inequality allows for dust absorption of ionizing photons. Using the $9 \mathrm{GHz}$ spectral luminosities of our sources in Sample A (Section 4) for $L_{\nu \text {,thermal }}$ and adopting an electron temperature of $T_{e}=10^{4} \mathrm{~K}$, the ionizing luminosities would be in the range $\log Q_{\mathrm{Lyc}} \sim 51.4-54.9$ (median value of $\log$ $Q_{\mathrm{Lyc}} \sim 52.5$ ), under the assumption that the radio emission is thermal. We estimate the number of massive stars that would be necessary to power this ionizing radiation using the results from Vacca et al. (1996); a "typical" O-type star (type O7.5 V) produces $Q_{\mathrm{Lyc}}=10^{49} \mathrm{~s}^{-1}$. Therefore, approximately 260 to $7.5 \times 10^{5}$ typical O-type stars would be necessary to account for the observed radio emission in our sources.

For comparison, we consider the Galactic thermal radio source W49A (Mezger et al. 1967), which is one of the youngest and most luminous star-forming regions in the Milky Way, as well as known extragalactic young massive star clusters. We calculate a $9 \mathrm{GHz}$ luminosity density of $L_{9 \mathrm{GHz}}=$ $10^{18.0} \mathrm{~W} \mathrm{~Hz}^{-1}$ for W49A using a flux density of $65 \mathrm{Jy}$ from Mezger et al. (1967) and a distance of $11.4 \mathrm{kpc}$ from Gwinn et al. (1992). Using Equation (1) yields an ionizing luminosity of $\log Q_{\mathrm{Lyc}} \sim 50.9$, or the equivalent of 79 typical O-type stars. Figure 5 (left panel) shows that the compact radio sources in our sample are orders of magnitude more luminous than the Galactic massive star-forming region W49A. H II regions produced by extragalactic young massive star clusters can also be identified as compact free-free (thermal) radio sources (e.g., Kobulnicky \& Johnson 1999; Turner et al. 2000; Reines et al. 2008; Johnson et al. 2009; Aversa et al. 2011; Kepley et al. 2014), yet even the most extreme sources have log $Q_{\text {Lyc }} \lesssim 10^{53}$ (Turner et al. 2000; Johnson et al. 2009).

We estimate the instantaneous SFRs from the ionizing luminosities calculated above for our sample following Kennicutt (1998):

$$
\operatorname{SFR}\left(M_{\odot} \mathrm{yr}^{-1}\right)=1.08 \times 10^{-53} Q_{\mathrm{Lyc}}\left(\mathrm{s}^{-1}\right)
$$

and compare these results to the SFRs of the entire host galaxies derived from GALEX far-ultraviolet (FUV) magnitudes from the NSA and WISE $22 \mu \mathrm{m}$ data from the AllWISE Source Catalog. We calculate the host galaxy dust-corrected SFRs following 

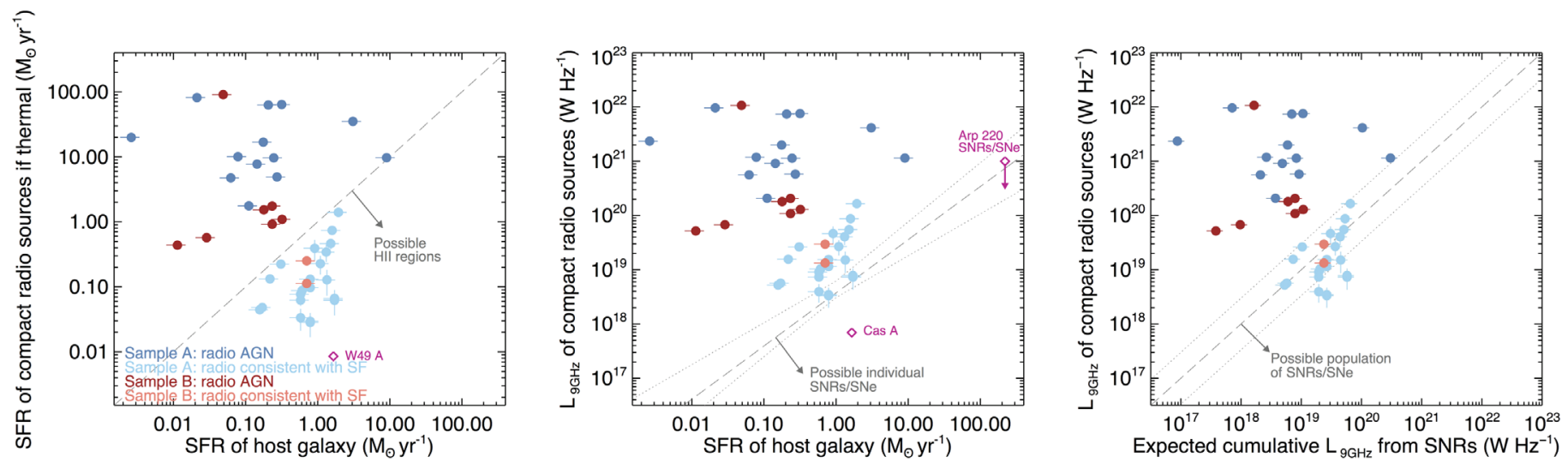

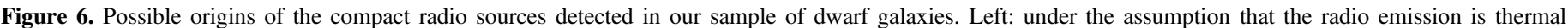

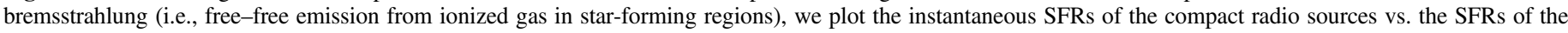

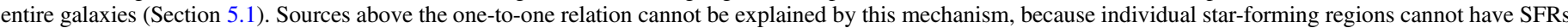

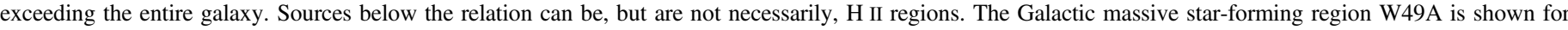

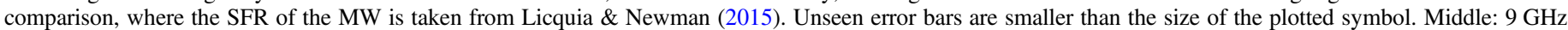

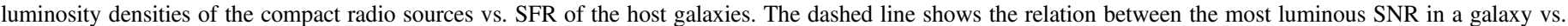

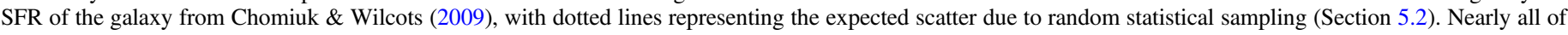

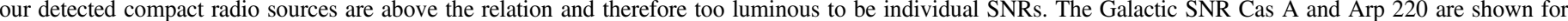

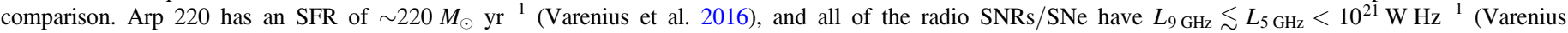

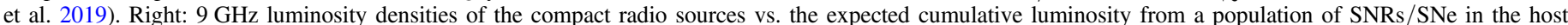
galaxies (Section 5.3). The dashed line indicates the one-to-one relation, and the dotted lines are offset by a factor of three.

Kennicutt \& Evans (2012) and Hao et al. (2011):

$$
\log \mathrm{SFR}=\log L(\mathrm{FUV})_{\mathrm{corr}}-43.35,
$$

where

$$
L(\mathrm{FUV})_{\mathrm{corr}}=L(\mathrm{FUV})_{\mathrm{obs}}+3.89 L(25 \mu \mathrm{m})
$$

and luminosities are in units of erg $\mathrm{s}^{-1}$. The $1 \sigma$ uncertainty using this method is $\sim 0.13 \mathrm{dex}$ (Hao et al. 2011). We estimate $L(25 \mu \mathrm{m})$ from the WISE $22 \mu \mathrm{m}$ data because the flux density ratio for these bands is expected to be on the order of unity for both late-type and early-type galaxies (Jarrett et al. 2013). Nine of the galaxies are not detected at $22 \mu \mathrm{m}$; in these cases, we derive SFRs using the uncorrected observed FUV luminosities.

Under the assumption that the compact radio emission is thermal, 13 of the 35 compact radio sources in Sample A would have SFRs larger than the SFRs of their entire host galaxies (see left panel of Figure 6). ${ }^{11}$ Clearly, this is highly unlikely, enabling us to reasonably rule out thermal bremsstrahlung as the origin of the radio emission for these objects. The calculated SFRs (assuming the compact radio emission is thermal) are $\sim 2$ to $81 M_{\odot} \mathrm{yr}^{-1}$, which is up to two orders of magnitude higher than the SFRs of well-studied extragalactic $\mathrm{H}$ II regions as noted above.

The implied SFR surface densities, $\Sigma_{\text {SFR }}$, of these 13 compact radio sources (if thermal) are also extraordinarily high, ranging from $\Sigma_{\mathrm{SFR}} \sim 320-4100 M_{\odot} \mathrm{yr}^{-1} \mathrm{kpc}^{-2}$. All of the sources would have $\Sigma_{\text {SFR }}$ greater than the "starburst intensity limit" of $45 M_{\odot} \mathrm{yr}^{-1} \mathrm{kpc}^{-2}$ proposed by Meurer et al. (1997). They would also be higher than all 61 of the normal spiral galaxies and all but one (Arp 220) of the 36 IR-selected circumnuclear starburst galaxies in the compilation of Kennicutt (1998). Given all of the findings above, it is highly unlikely that star-forming regions producing free-free radiation can fully account for the compact radio sources detected in our sample of dwarf galaxies.

\footnotetext{
11 We note that the dark blue point toward the right (ID 92) is consistent with falling on the one-to-one relation when uncertainties are taken into account.
}

\subsection{Individual SNRs/SNe?}

We also consider whether individual SNRs or younger SNe could plausibly account for the compact radio emission observed in our sample of dwarf galaxies. First, we compare the $9 \mathrm{GHz}$ spectral luminosities of our detected sources with that of Cas A, which is one of the youngest and most luminous SNRs in the Milky Way, with an age of $~ 300$ yr. Adopting a $1 \mathrm{GHz}$ flux density of $S_{1 \mathrm{GHz}}=2723 \mathrm{Jy}$ and a spectral index of $\alpha=-0.770$ from Baars et al. (1977) (epoch 1980.0), and distance of $3.4 \mathrm{kpc}$ from Reed et al. (1995), we calculate a $9 \mathrm{GHz}$ spectral luminosity of $L_{9 \mathrm{GHz}} \sim 7 \times 10^{17} \mathrm{~W} \mathrm{~Hz}^{-1}$ for Cas A. Figure 5 shows that our detected compact radio sources are orders of magnitude more luminous than Cas A.

Younger radio supernovae ( $\mathrm{SNe}$ ) have typical ages less than a few decades. These radio sources can be significantly more luminous than older SNRs, albeit for a relatively short amount of time. During the SN stage, the blast wave from the explosion is thought to expand in a dense circumstellar medium, whereas in the SNR stage, the blast wave is thought to interact with the surrounding interstellar medium. Varenius et al. (2019) and Ulvestad (2009) study the populations of radio SNe/SNRs in the luminous infrared merging galaxies Arp 220 and Arp 299, respectively. The $5 \mathrm{GHz}$ luminosity densities of 88 sources in Arp 220 are shown in Figure 6 of Varenius et al. (2019) and the 8.4 GHz luminosity densities of 14 sources in Arp 299 are given in Figure 7 of Ulvestad (2009). The vast majority of the radio $\mathrm{SNe}$ in these works have luminosity densities $L_{\nu} \lesssim 10^{20} \mathrm{~W} \mathrm{~Hz}{ }^{-1}$, with the brightest sources having $L_{\nu}<10^{21} \mathrm{~W} \mathrm{~Hz}^{-1}$. We consider this an upper limit on $L_{9 \mathrm{GHz}}$ for these sources since SNe/SNRs typically have steep radio spectra that decrease with increasing frequency. Nine of the compact radio sources in our Sample A have luminosity densities higher than all of $\sim 100 \mathrm{SNe} / \mathrm{SNRs}$ found in Arp 220 and Arp 299.

To more robustly determine if individual SNRs/SNe could produce the observed radio emission, we make use of the relation between the luminosity density of the brightest 

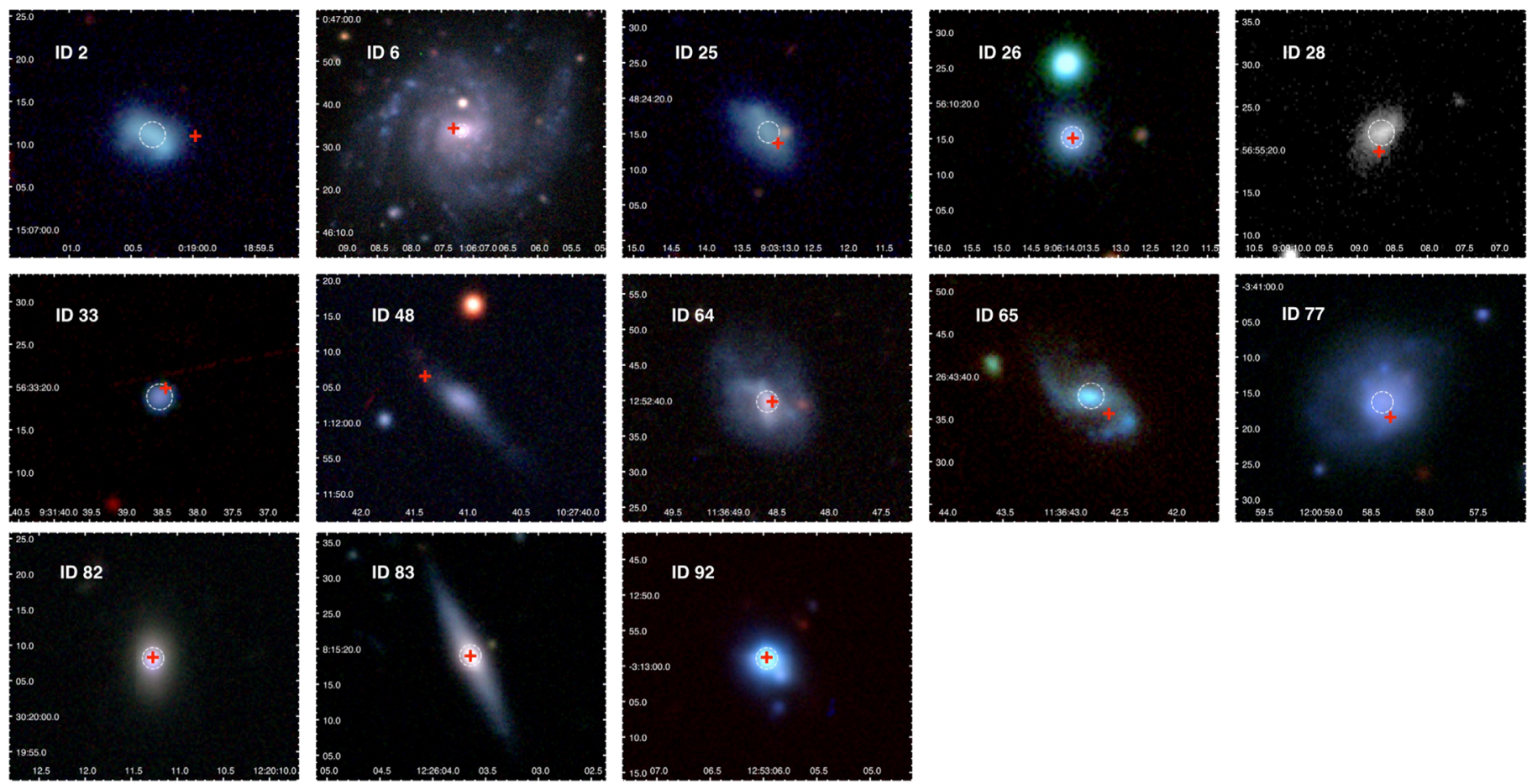

Figure 7. DECaLS grz-band images of dwarf galaxies (in Sample A) with the strongest evidence for accreting massive BHs (see Section 5.4). ID 28 only has a $z$-band image, which is shown here in grayscale. Red crosses indicate the positions of the compact radio sources, and the white dashed circles indicate the positions of the SDSS spectroscopic fibers (with diameters of $3^{\prime \prime}$ ). ID 48 does not have an SDSS spectrum. Absolute astrometry for the optical and radio images is accurate to $\lesssim 0$ " 1 and uncertainties in the radio source centroids are also $\lesssim 0$ !' 1 .

$\mathrm{SNR} / \mathrm{SNe}$ in a galaxy and the SFR of the galaxy given by Chomiuk \& Wilcots (2009):

$$
L_{1.4}^{\max }=\left(95_{-23}^{+31}\right) \mathrm{SFR}^{0.98 \pm 0.12},
$$

where the $1.4 \mathrm{GHz}$ luminosity density is in units of $10^{24} \mathrm{erg} \mathrm{s}^{-1}$ $\mathrm{Hz}^{-1}$ and the SFR is in units of $M_{\odot} \mathrm{yr}^{-1}$. We scale this relation to the luminosity density at $9 \mathrm{GHz}$, assuming a spectral index of $\alpha=-0.5$ (Chomiuk \& Wilcots 2009), and plot it with our measurements of $L_{9 \mathrm{GHz}}$ (Section 4) versus galaxy SFR (Section 5.1) in the middle panel of Figure 6. Nearly all of our detected radio sources lie above the relation (including scatter), indicating that individual SNRs/SNe are not a viable explanation for the compact radio sources detected in our sample of dwarf galaxies.

\subsection{Populations of SNRs/SNe?}

We also consider multiple SNRs/SNe as a possible origin for the compact radio emission, making use of the radio SNR luminosity function derived from a sample of 19 nearby galaxies spanning the SMC to Arp 220 (Chomiuk \& Wilcots 2009). We adopt the luminosity function that is proportional to the host galaxy SFR, although we note that a population of SNRs/SNe producing the detected radio emission would need to be confined to roughly the size of the VLA beam, as the majority of our detections are point-like at a resolution of $\sim 0$ ". 25 (corresponding to a physical scale of $\sim 85 \mathrm{pc}$ at the median distance of our sample). The luminosity function is given by

$$
n(L)=\frac{d N}{d L}=92 \times \mathrm{SFR} \times L^{-2.07}
$$

where $n(L)$ is the number of SNRs with $1.4 \mathrm{GHz}$ luminosity density $L$, and the SFRs of our sample galaxies are determined in Section 5.1. The total expected luminosity from all SNRs in a given galaxy is then

$$
L_{\text {total }}=\int n(L) L d L
$$

where we take the integral from 0.1 to $10^{4}$ to span the full range of individual SNRs/SNe presented in Chomiuk \& Wilcots (2009). For each galaxy in our sample, we convert $L_{\text {total }}$ (in units of $10^{24} \mathrm{erg} \mathrm{s}^{-1} \mathrm{~Hz}^{-1}$ ) to a luminosity at $9 \mathrm{GHz}$ (in $\mathrm{W} \mathrm{Hz}^{-1}$ ) assuming a spectral index of $\alpha=-0.5$ (Chomiuk $\&$ Wilcots 2009). A comparison between the expected galaxywide luminosity of all SNRs and our detected compact radio sources is shown in the right panel of Figure 6. Thirteen radio sources in Sample A have luminosities more than a factor of three above the expected cumulative contribution from all SNRs/SNe in their respective host galaxies, indicating that an alternative origin for the radio emission is required.

\subsection{AGNs}

Based on the analysis above, we have identified 13 compact radio sources in Sample A that are almost certainly AGNs. Figure 7 shows optical images of the host galaxies from the Dark Energy Camera Legacy Survey (DECaLS). The compact radio sources toward these dwarf galaxies are inconsistent with thermal $\mathrm{H}$ II regions, individual SNRs/SNe, or populations of SNRs/SNe. One of these objects, ID 26, was previously identified as a broad-line AGN (designated RGG 9) in Reines et al. (2013) with $M_{\mathrm{BH}} \sim 2.5 \times 10^{5} M_{\odot}$. Additional compact radio sources detected in the other dwarf galaxies in Sample A 

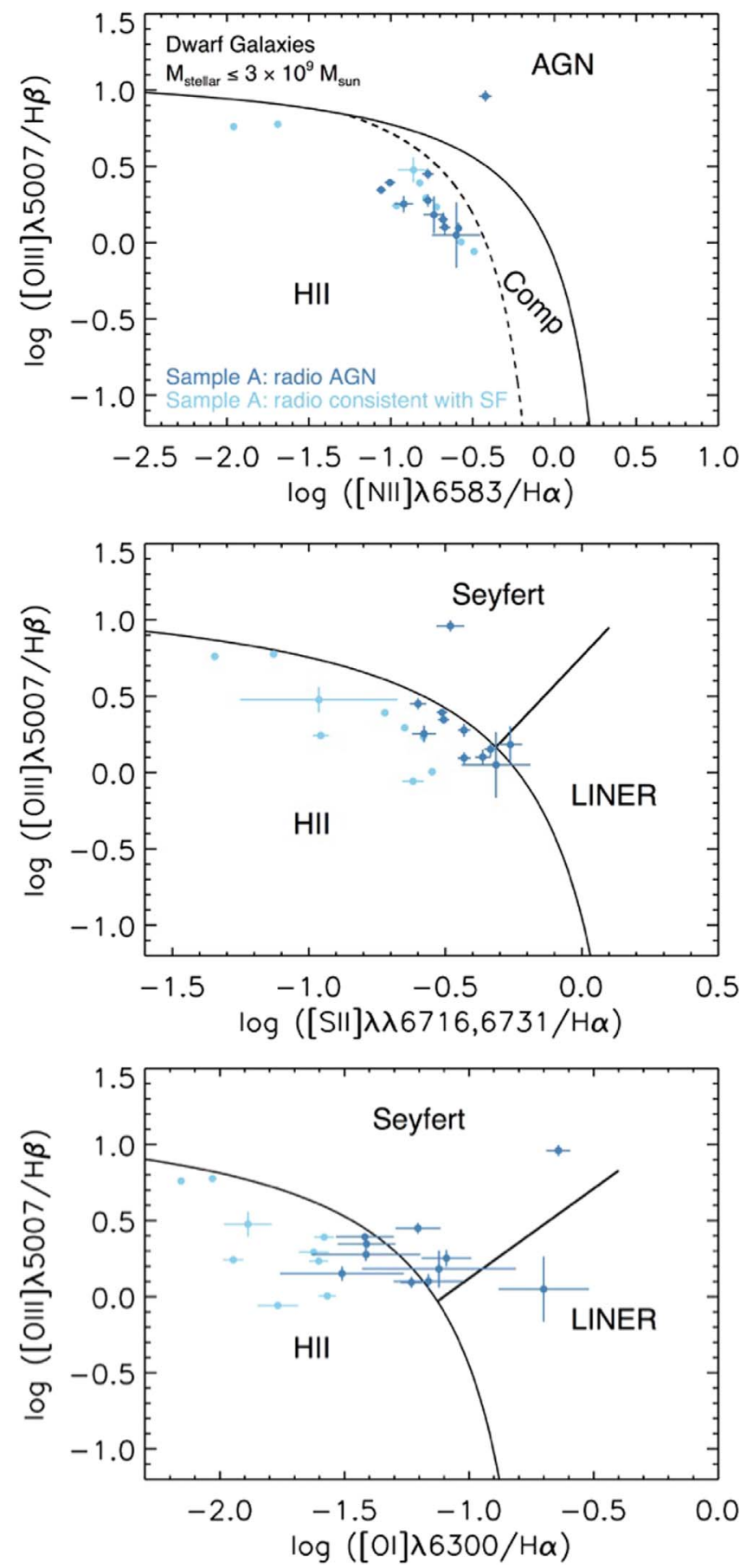

Figure 8. Optical emission line diagnostic diagrams for the galaxies in Sample A with SDSS spectra and emission line measurements from Reines \& Volonteri (2015). Galaxies in Sample B do not have SDSS spectra. The demarcations come from the classification scheme outlined in Kewley et al. (2006).

may also be AGNs; however, we cannot reliably rule out alternative explanations. We discuss the objects in Sample B in Section 5.4.1.

We investigate where the host galaxies in Sample A fall on optical diagnostic diagrams for those with SDSS spectra and narrow emission line measurements by Reines \& Volonteri (2015). None of the galaxies in Sample B have SDSS spectra. The narrow line diagnostic diagrams are shown in Figure 8. ID 26 (RGG 9) is the only dwarf galaxy with emission line ratios clearly dominated by an AGN in all three diagrams. The other sources fall in the star-forming region of the BPT diagram (Baldwin et al. 1981), which takes [O III] $/ \mathrm{H} \beta$ versus [N II] $/ \mathrm{H} \alpha$. However, five objects (IDs 25, 26, 64, 65, 83) fall in the Seyfert region of the $[\mathrm{O} \mathrm{III}] / \mathrm{H} \beta$ versus $[\mathrm{O} \mathrm{I}] / \mathrm{H} \alpha$ diagram, and one other object falls in the LINER region (ID 6). These seemingly contradictory classifications do not preclude the existence of AGNs, as $[\mathrm{O} \mathrm{I}] / \mathrm{H} \alpha$ is sensitive to the hardness of the radiation field, which moves AGN host galaxies to the right, while $[\mathrm{N} \mathrm{II}] / \mathrm{H} \alpha$ is primarily sensitive to metallicity and low-mass galaxies are generally metal-poor, which moves them to the left (e.g., Groves et al. 2006; Kewley et al. 2006). We also note that the radio sources do not always fall within the SDSS spectroscopic fiber (see Figure 7), so these optical classifications should be taken with caution.

Many of the radio-selected AGNs are not located in welldefined galaxy nuclei (Figures 7 and 9), in contrast to the optically selected AGNs in dwarf galaxies found by Reines et al. (2013). Roughly half of the galaxies have radio sources with offsets $\gtrsim 2^{\prime \prime}$ relative to the optical centers provided in the NSA. The absolute astrometry errors on both the optical and radio positions are $\lesssim 0$ " 1 . Some of the host dwarf galaxies do not even possess obvious photometric centers, having irregular morphologies and/or showing signs of interactions/mergers.

We therefore carefully consider whether the 13 AGNs in Sample A reside in the target dwarf galaxies or are spurious background sources (also see Sections 2.1 and 4). Up to $\sim 7$ background interlopers could be present, but there is compelling evidence that the radio AGNs in Sample A are indeed associated with the target galaxies. First, unlike the background sources highlighted in Figure 3, the off-nuclear radio sources in Sample A lack detectable optical counterparts. This argues against background interlopers, as they would have to be very obscured (particularly at DECaLS depths) or at high redshift.

Additionally, Figure 10 (left panel) shows that the dwarf galaxies in Sample A with radio-selected AGNs have systematically higher ratios of $[\mathrm{O} \mathrm{I}] / \mathrm{H} \alpha$ relative to the other dwarfs in Sample A that have less luminous radio sources consistent with star formation. Combined with the aforementioned Seyfert/LINER classifications, this suggests that the radio sources are likely associated with the galaxies that are producing the optical line emission, rather than background interlopers.

We also see a trend such that the more extended/disturbed (later-type) galaxies tend to have more offset radio sources, while the centrally concentrated (earlier-type) galaxies tend to have nuclear radio sources (see Figure 7). This is quantified in the right panel of Figure 10, which shows the radio-optical positional offset versus (inverse) concentration index defined as $C=r_{50} / r_{90}$, where $r_{50}$ and $r_{90}$ are the half-light and $90 \%$ light Petrosian radii, respectively. In other words, the radio sources tend to fall in the nucleus of nucleated dwarf galaxies, whereas the radio sources are more offset for those galaxies without prominent nuclei. This is consistent with simulations that predict a population of "wandering" BHs in dwarf galaxies, primarily due to interactions/mergers (Bellovary et al. 2019).

\subsubsection{Additional Objects in Sample B Including a Candidate Dual AGN}

We detect radio AGNs in six additional galaxies in Sample B (Figure 11). SDSS spectra are not available for the galaxies in Sample B, and because we do not have reliable spectroscopic 


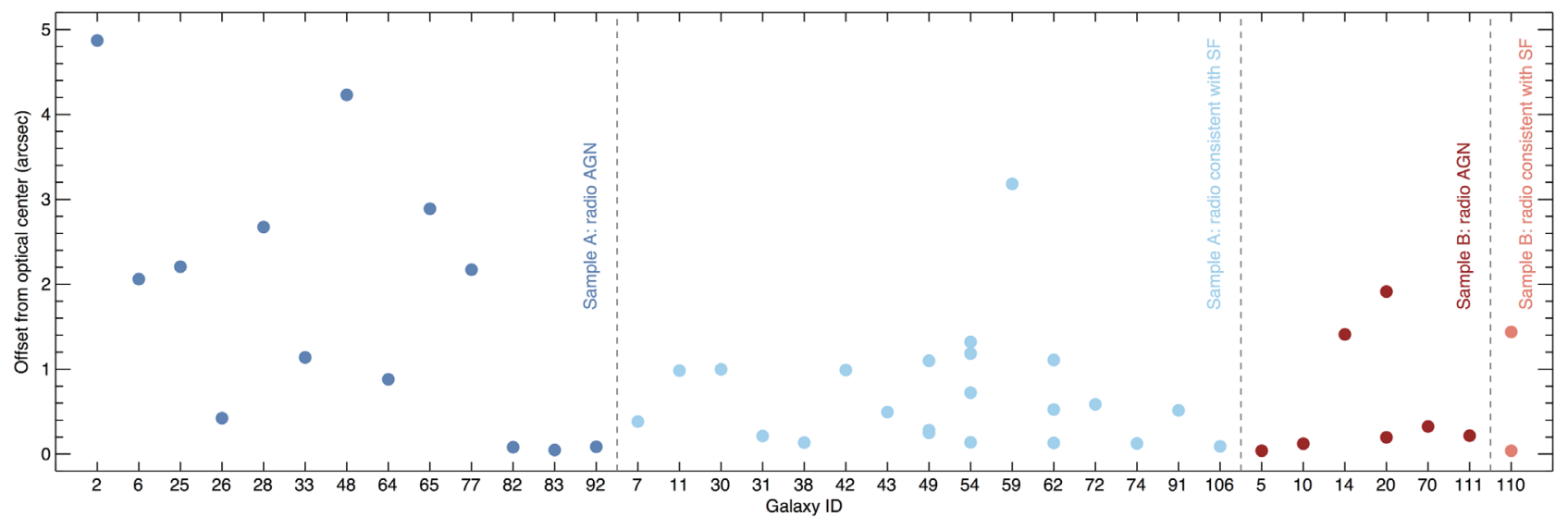

Figure 9. Offset of radio sources relative to the optical centers of the target galaxies. The SDSS, as well as the VLA observations, have absolute astrometry accurate to $\lesssim 00^{\prime \prime} 1$.
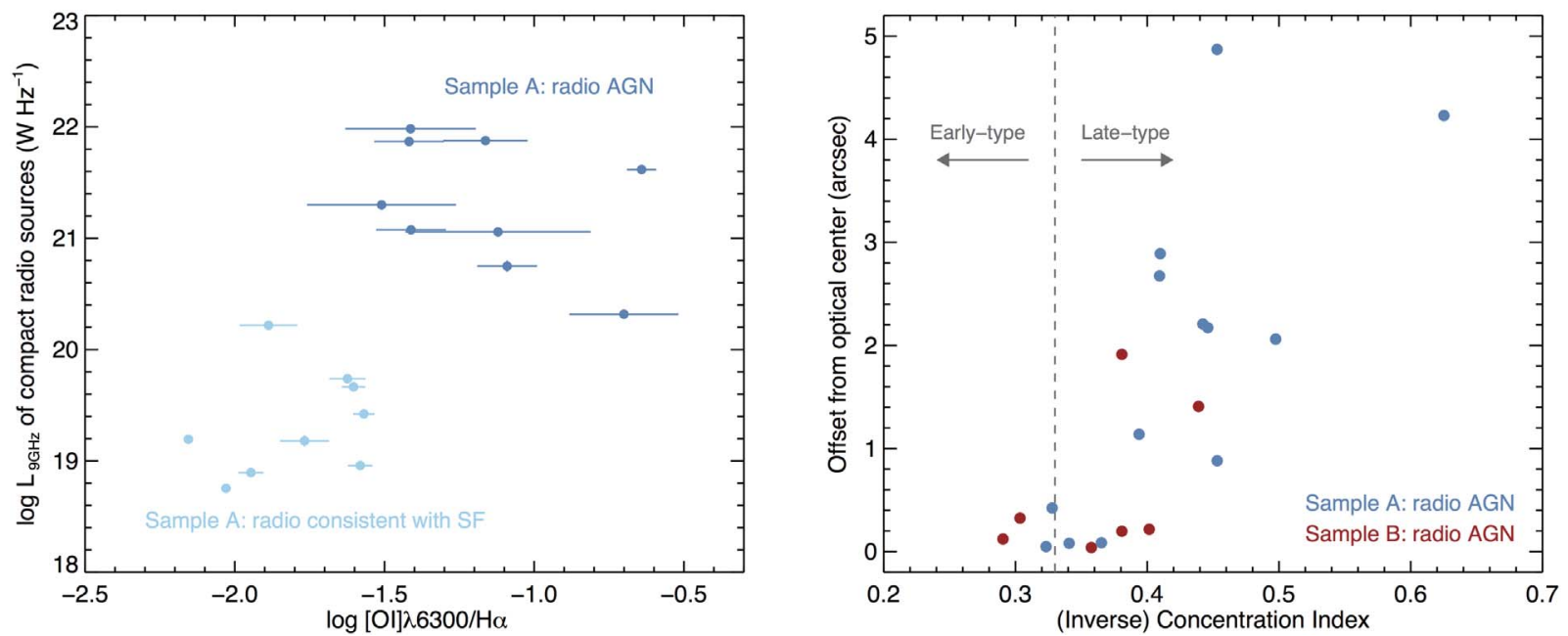

Figure 10. Left: luminosity density at $9 \mathrm{GHz}$ vs. [O I]/H $\alpha$ for dwarf galaxies in Sample A with SDSS spectra and emission line measurements from Reines \& Volonteri (2015). The radio AGNs have systematically higher [O I]/H $\alpha$ ratios, suggesting the radio sources are associated with the dwarf galaxies producing the optical line emission, rather than background interlopers. Right: offset of radio source from optical center of galaxy vs. (inverse) concentration index defined as $C=r_{50} / r_{90}$ (Shimasaku et al. 2001). The more centrally concentrated galaxies have radio AGNs near their centers, while the more extended/disturbed galaxies tend to host offset radio AGNs.

redshifts, the distances and masses are uncertain for these galaxies. Moreover, we cannot investigate where they fall on the optical diagnostic diagrams.

Photometric redshifts are available from the SDSS DR14 Sky Server and fall in the range $0.08 \lesssim z_{\text {phot }} \lesssim 0.16$ (with a median value of 0.1 ). These values are significantly larger than the redshifts in the NSA $\left(0.005 \lesssim z_{\text {phot }} \lesssim 0.016\right.$, median value of 0.01). Along with the redder colors relative to the dwarf galaxies in Sample A (see Figure 11), this suggests these galaxies are much more massive than the values provided in the NSA $\left(M_{\text {stellar }} \lesssim 2 \times 10^{9} M_{\odot}\right)$.

We detect two radio AGNs toward ID 20. Figure 12 shows a DECaLS image of the system, which appears to consist of two galaxies. The radio sources we detect are approximately 1"!7 apart and coincide with the optical galaxies. Under the assumption that galaxies are at the same redshift $\left(z_{\text {phot }} \sim 0.16\right)$, this corresponds to a physical projected separation of $\sim 5.5 \mathrm{kpc}$. Therefore, ID 20 is a strong dual AGN candidate (e.g.,
Comerford et al. 2015), one of only a handful of those detected in the radio (e.g., Fu et al. 2015; Müller-Sánchez et al. 2015).

\section{Properties of the Dwarf Galaxies Hosting Radio-selected AGNs}

We obtain galaxy half-light radii, total stellar masses, and colors from the NSA (Table 1). The galaxies in Sample A with radio-selected AGNs are physically small. The half-light radii span a range of $\sim 0.5-10 \mathrm{kpc}$, with the majority (10/13) having $r_{50} \lesssim 3 \mathrm{kpc}$. Total stellar masses are in the range $M_{\star} \sim 10^{7.8}-10^{9.4} M_{\odot}$ with a median value of $M_{\star} \sim 10^{9.2} M_{\odot}$. Three of the galaxies have stellar masses below $M_{\star} \lesssim 3 \times$ $10^{8} M_{\odot}$, which is the mass of the lowest-mass BPT AGN from Reines et al. (2013) and equivalent to the stellar mass of the SMC (Stanimirović et al. 2004). The radio-detected dwarf galaxies follow the overall color distribution of the general population of dwarf galaxies, whereas the optically selected sample of AGNs in dwarfs from Reines et al. (2013) tend to have redder host galaxies 
Radio AGN

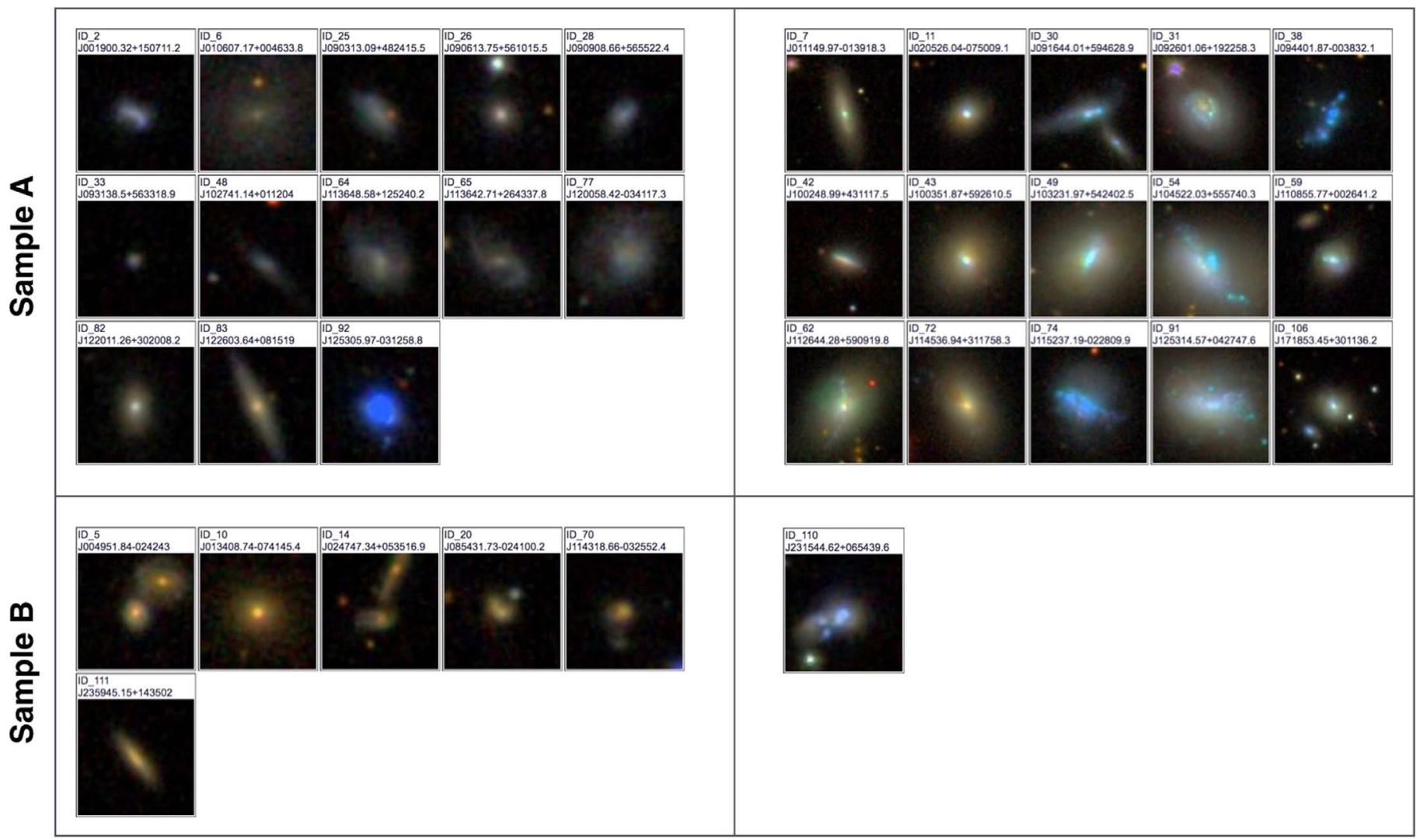

Figure 11. SDSS images of the galaxies with compact radio sources detected in our VLA observations. All of the compact radio sources are within $5^{\prime \prime}$ of the optical center of the galaxy. See Table 1 for galaxy properties. Images are $25^{\prime \prime}$ on a side, except the ones with radio sources consistent with star formation, which are $50^{\prime \prime}$ on a side.

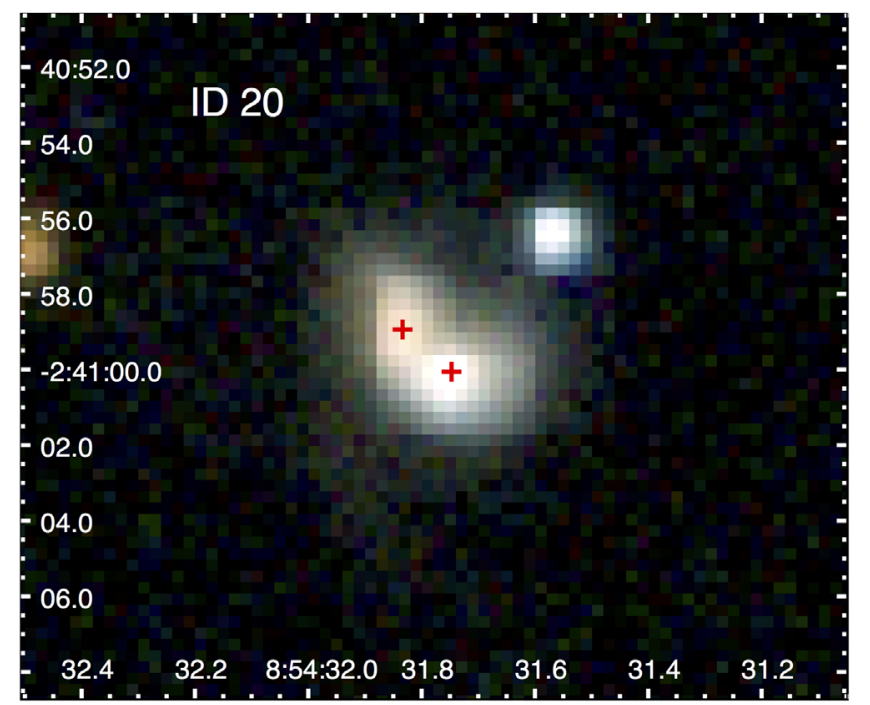

Figure 12. DECaLS image of the dual radio AGN candidate ID 20. Red crosses indicate the positions of the VLA sources.

(see Figure 13). This illustrates the advantage of using highresolution radio observations to overcome some of the bias against finding AGNs in bluer star-forming dwarf galaxies that optical selection suffers.

Mid-infrared colors of the galaxies in our sample are shown in Figure 14. We use the WISE magnitudes from the AllWISE Source Catalog to see if any of the sources show signs of

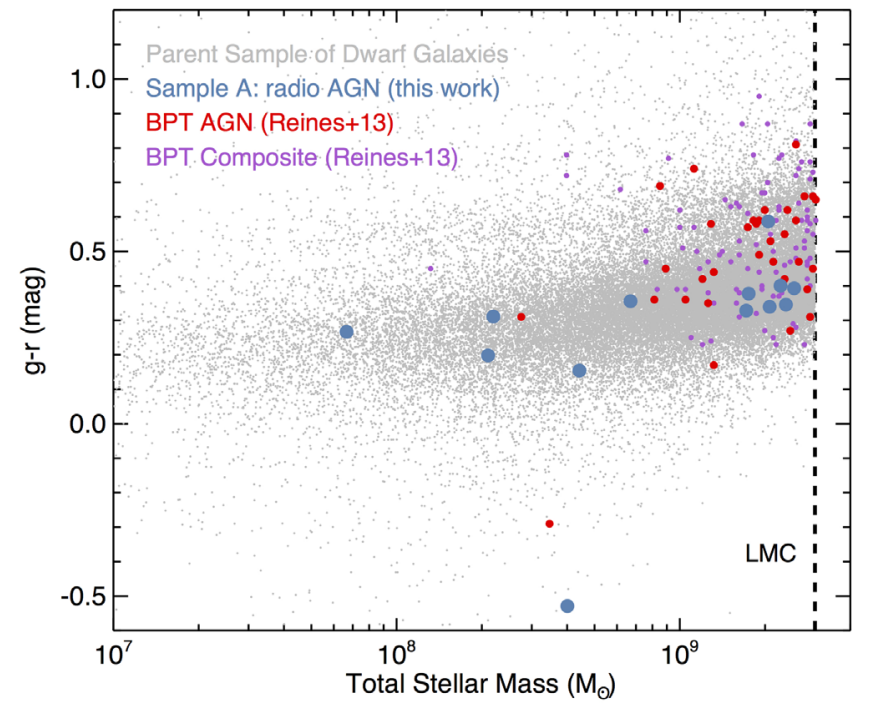

Figure 13. Plot of $g-r$ color vs. total stellar mass for dwarf galaxies with $M_{\star} \leqslant 3 \times 10^{9} M_{\odot}$ in the NSA. The radio-detected AGNs in this work are shown along with the optically selected AGNs and composites from Reines et al. (2013).

AGN-heated dust. ID 26 (RGG 9) is the only dwarf galaxy that falls in the Jarrett et al. (2011) AGN selection box. The majority of the objects in Sample A are dominated by infrared emission from the host galaxies. A couple of additional objects would be selected by the Stern et al. (2012) AGN selection 


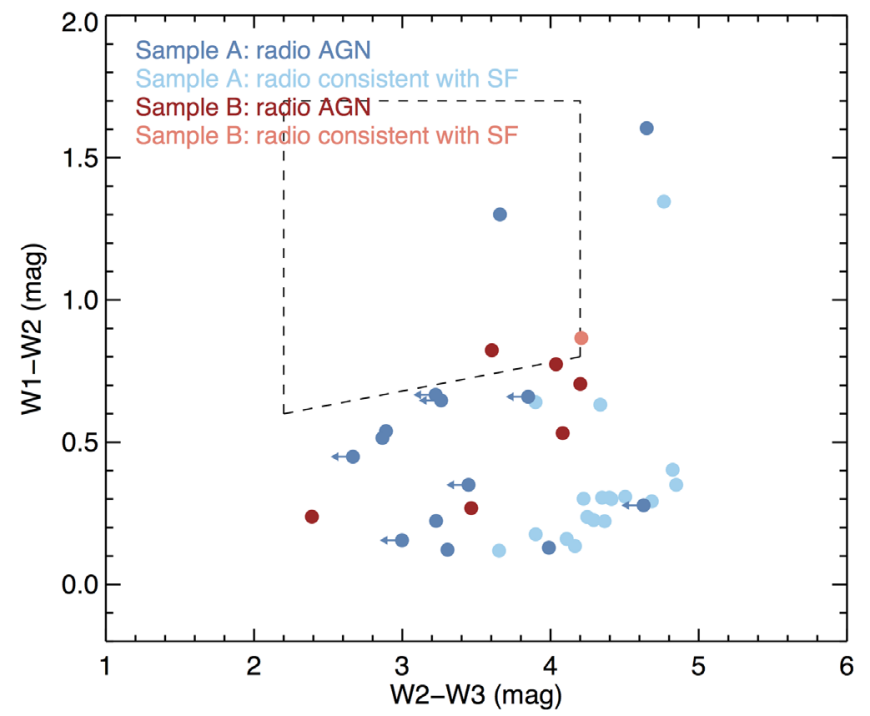

Figure 14. WISE color-color diagram for the galaxies in our sample. The Jarrett et al. (2011) AGN selection box is shown with dashed lines.

criterion, $W 1-W 2 \geqslant 0.8$. While this single color selection has proved useful for luminous sources, Hainline et al. (2016) caution that dwarf starburst galaxies can severely contaminate samples at low stellar masses.

\section{Conclusions and Discussion}

Here, we present the first large-scale radio search for massive BHs in nearby dwarf galaxies $\left(M_{\star} \lesssim 3 \times 10^{9} M_{\odot}\right.$; also see Mezcua et al. (2019) for a search at intermediate-redshifts using VLA-COSMOS). We observe 111 dwarf galaxies detected in the FIRST survey using new VLA continuum observations at $X$ band with $\sim 10$ times the sensitivity and a beam solid angle that is smaller by a factor of $\sim 400$, compared to FIRST. Thirteen dwarf galaxies have compact radio sources that are almost certainly AGNs, as they are inconsistent with alternative explanations for the radio emission (e.g., thermal H II regions, individual SNRs/SNe, populations of $\mathrm{SNRs} / \mathrm{SNe}$ ). We also find evidence suggesting the radio AGNs are indeed associated with the dwarf galaxies, rather than background interlopers (e.g., Figure 10).

Overall, the host galaxies are bluer than dwarfs with optically selected AGNs (Reines et al. 2013), they have narrow emission line ratios falling in the star-forming region of the $[\mathrm{O}$ III] $/ \mathrm{H} \beta$ versus $[\mathrm{N} \mathrm{II}] / \mathrm{H} \alpha$ BPT diagram, and they have midIR colors dominated by stellar emission. With one exception (ID 26, RGG 9), the AGNs we have found in dwarf galaxies using high-resolution radio observations are effectively hidden at optical/IR wavelengths.

The $\mathrm{BH}$ masses in these systems are largely unknown. However, if we assume the $\mathrm{BH}$ mass to total stellar mass relation derived from optically selected broad-line AGNs (Reines \& Volonteri 2015), we would expect BH masses in the range of $M_{\mathrm{BH}} \sim 10^{4.1}-10^{5.8} M_{\odot}$, with a median value of $M_{\mathrm{BH}} \sim 10^{5.6} M_{\odot}$. We caution that there are large uncertainties associated with these mass estimates. The scatter in the Reines \& Volonteri (2015) AGN relation is 0.55 dex, and it is not clear that this relation is applicable to our host galaxies-particularly for any galaxies undergoing interactions/mergers.

Many of the radio AGNs we identify are not located in welldefined nuclei, and some are significantly offset from the center of their host galaxy (Figure 7). This is distinct from the known dwarf galaxies hosting optically selected AGNs (e.g., Reines et al. 2013; Schutte et al. 2019). While surprising from an observational standpoint, recent simulations have predicted that roughly half of all massive BHs in dwarf galaxies are expected to be wandering around in the outskirts of their host galaxies (Bellovary et al. 2019). Off-nuclear BHs may have been tidally stripped during mergers (e.g., Governato et al. 1994; Bellovary et al. 2010; Tremmel et al. 2015), or they could be recoiling merged BHs (e.g., Merritt et al. 2004; Volonteri \& Perna 2005). The coalescence of two BHs will produce gravitational waves that carry momentum, causing the merged black hole to undergo a velocity kick and spatial displacement. Gravitational waves from massive $\mathrm{BH}$ mergers in dwarf galaxies are expected to be detectable with future observations with LISA. Once a massive black hole has left the center of a dwarf galaxy, it is unlikely to return. This is in contrast to more massive galaxies where dynamical friction is more efficient and can bring the black hole back to the nucleus.

We are currently pursuing a broad range of follow-up observations of our sample. For example, spatially resolved spectroscopy covering the positions of the radio sources will help confirm that they are not background objects, and VLBI observations may resolve the radio emission or confine it to a more compact core. Additionally, high-resolution X-ray observations will help solidify the AGN nature of the radio sources, and it can be used with the fundamental plane of $\mathrm{BH}$ activity to estimate $\mathrm{BH}$ masses and Eddington ratios.

Finally, this work highlights the potential of deep, highresolution radio observations for searching for (wandering) massive BHs in dwarf galaxies. Ultimately we need a larger, unbiased radio survey (e.g., with a next generation VLA; see Plotkin \& Reines 2018) to better constrain the occupation fraction of massive BHs in dwarf galaxies and thereby determine the mechanism that seeded the first high-redshift BHs.

A.E.R. is extremely grateful to Amy Kimball, Jürgen Ott, and Drew Medlin at NRAO in Socorro for helping with the VLA data reduction. The authors also thank Andy Goulding for helpful discussions about galaxy ID 47 and the referee for a constructive report. The National Radio Astronomy Observatory is a facility of the National Science Foundation operated under cooperative agreement by Associated Universities, Inc. This publication makes use of data products from the Widefield Infrared Survey Explorer, which is a joint project of the University of California, Los Angeles, and the Jet Propulsion Laboratory/California Institute of Technology, funded by NASA. This work has also used observations made with the NASA Galaxy Evolution Explorer. GALEX is operated for NASA by the California Institute of Technology under NASA contract NAS5-98034. This study has made use of the NASA/ IPAC Extragalactic Database (NED), which is operated by the Jet Propulsion Laboratory, California Institute of Technology, under contract with NASA. We are grateful to Michael Blanton and all who helped create the NASA-Sloan Atlas. Funding for the NASA-Sloan Atlas has been provided by the NASA Astrophysics Data Analysis Program (08-ADP08-0072) and the NSF (AST-1211644).

Funding for the Sloan Digital Sky Survey IV has been provided by the Alfred P. Sloan Foundation, the U.S. Department of Energy Office of Science, and the Participating 
Institutions. SDSS-IV acknowledges support and resources from the Center for High-Performance Computing at the University of Utah. The SDSS website is www.sdss.org. SDSS-IV is managed by the Astrophysical Research Consortium for the Participating Institutions of the SDSS Collaboration including the Brazilian Participation Group, the Carnegie Institution for Science, Carnegie Mellon University, the Chilean Participation Group, the French Participation Group, Harvard-Smithsonian Center for Astrophysics, Instituto de Astrofísica de Canarias, The Johns Hopkins University, Kavli Institute for the Physics and Mathematics of the Universe (IPMU)/University of Tokyo, the Korean Participation Group, Lawrence Berkeley National Laboratory, Leibniz Institut für Astrophysik Potsdam (AIP), Max-Planck-Institut für Astronomie (MPIA Heidelberg), Max-Planck-Institut für Astrophysik (MPA Garching), Max-Planck-Institut für Extraterrestrische Physik (MPE), National Astronomical Observatories of China, New Mexico State University, New York University, University of Notre Dame, Observatário Nacional/MCTI, The Ohio State University, Pennsylvania State University, Shanghai Astronomical Observatory, United Kingdom Participation Group, Universidad Nacional Autónoma de México, University of Arizona, University of Colorado Boulder, University of Oxford, University of Portsmouth, University of Utah, University of Virginia, University of Washington, University of Wisconsin, Vanderbilt University, and Yale University.

The Legacy Surveys consist of three individual and complementary projects: the Dark Energy Camera Legacy Survey (DECaLS; NOAO Proposal ID \# 2014B-0404; PIs: David Schlegel and Arjun Dey), the Beijing-Arizona Sky Survey (BASS; NOAO Proposal ID \# 2015A-0801; PIs: Zhou $\mathrm{Xu}$ and Xiaohui Fan), and the Mayall z-band Legacy Survey (MzLS; NOAO Proposal ID \# 2016A-0453; PI: Arjun Dey). DECaLS, BASS and MzLS together include data obtained, respectively, at the Blanco telescope, Cerro Tololo InterAmerican Observatory, National Optical Astronomy Observatory (NOAO); the Bok telescope, Steward Observatory, University of Arizona; and the Mayall telescope, Kitt Peak National Observatory, NOAO. The Legacy Surveys project is honored to be permitted to conduct astronomical research on Iolkam Du'ag (Kitt Peak), a mountain with particular significance to the Tohono O'odham Nation. NOAO is operated by the Association of Universities for Research in Astronomy (AURA) under a cooperative agreement with the National Science Foundation. This project used data obtained with the Dark Energy Camera (DECam), which was constructed by the Dark Energy Survey (DES) collaboration. Funding for the DES Projects has been provided by the U.S. Department of Energy, the U.S. National Science Foundation, the Ministry of Science and Education of Spain, the Science and Technology Facilities Council of the United Kingdom, the Higher Education Funding Council for England, the National Center for Supercomputing Applications at the University of Illinois at Urbana-Champaign, the Kavli Institute of Cosmological Physics at the University of Chicago, Center for Cosmology and Astro-Particle Physics at the Ohio State University, the Mitchell Institute for Fundamental Physics and Astronomy at Texas A\&M University, Financiadora de Estudos e Projetos, Fundacao Carlos Chagas Filho de Amparo, Financiadora de Estudos e Projetos, Fundacao Carlos Chagas Filho de Amparo a Pesquisa do Estado do Rio de Janeiro, Conselho Nacional de Desenvolvimento Cientifico e
Tecnologico and the Ministerio da Ciencia, Tecnologia e Inovacao, the Deutsche Forschungsgemeinschaft, and the Collaborating Institutions in the Dark Energy Survey. The Collaborating Institutions are Argonne National Laboratory, the University of California at Santa Cruz, the University of Cambridge, Centro de Investigaciones Energeticas, Medioambientales y Tecnologicas-Madrid, the University of Chicago, University College London, the DES-Brazil Consortium, the University of Edinburgh, the Eidgenossische Technische Hochschule (ETH) Zurich, Fermi National Accelerator Laboratory, the University of Illinois at Urbana-Champaign, the Institut de Ciencies de l'Espai (IEEC/CSIC), the Institut de Fisica d'Altes Energies, Lawrence Berkeley National Laboratory, the Ludwig-Maximilians Universitat Munchen and the associated Excellence Cluster Universe, the University of Michigan, the National Optical Astronomy Observatory, the University of Nottingham, the Ohio State University, the University of Pennsylvania, the University of Portsmouth, SLAC National Accelerator Laboratory, Stanford University, the University of Sussex, and Texas A\&M University. BASS is a key project of the Telescope Access Program (TAP), which has been funded by the National Astronomical Observatories of China, the Chinese Academy of Sciences (the Strategic Priority Research Program "The Emergence of Cosmological Structures" Grant \# XDB09000000), and the Special Fund for Astronomy from the Ministry of Finance. The BASS is also supported by the External Cooperation Program of Chinese Academy of Sciences (Grant \# 114A11KYSB20160057), and Chinese National Natural Science Foundation (Grant \# 11433005). The Legacy Survey team makes use of data products from the Near-Earth Object Wide-field Infrared Survey Explorer (NEOWISE), which is a project of the Jet Propulsion Laboratory/California Institute of Technology. NEOWISE is funded by the National Aeronautics and Space Administration. The Legacy Surveys imaging of the DESI footprint is supported by the Director, Office of Science, Office of High Energy Physics of the U.S. Department of Energy under Contract No. DE-AC02-05CH1123, by the National Energy Research Scientific Computing Center, a DOE Office of Science User Facility under the same contract, and by the U.S. National Science Foundation, Division of Astronomical Sciences under Contract No. AST-0950945 to NOAO.

\section{Appendix}

\section{A.1. ID 47-Serendipitous Discovery of an Optical AGN in a Dwarf Galaxy}

We detect an off-nuclear radio source toward the dwarf galaxy J1022-0055 (ID 47) with a luminosity much too high to be consistent with stellar processes. The radio emission consists of a bright point source plus a fainter elongated jet-like structure (Figure 4), features commonly associated with accreting massive BHs. The radio source is located 4!"9 away from the center of the galaxy in the northeast direction, and has a point-like optical counterpart that can be seen in groundbased imaging (Figure 3).

SDSS spectra were obtained for both the off-nuclear source and the galaxy center. Based on the narrow emission lines, the offset source has a redshift of $z=0.04828 \pm 0.00003$ and the galaxy center has a redshift of $z=0.04853 \pm 0.00001$, indicating a radial velocity offset of $\Delta v=75 \mathrm{~km} \mathrm{~s}^{-1}$ with a statistical error of $10 \mathrm{~km} \mathrm{~s}^{-1}$. In other words, the gas producing 

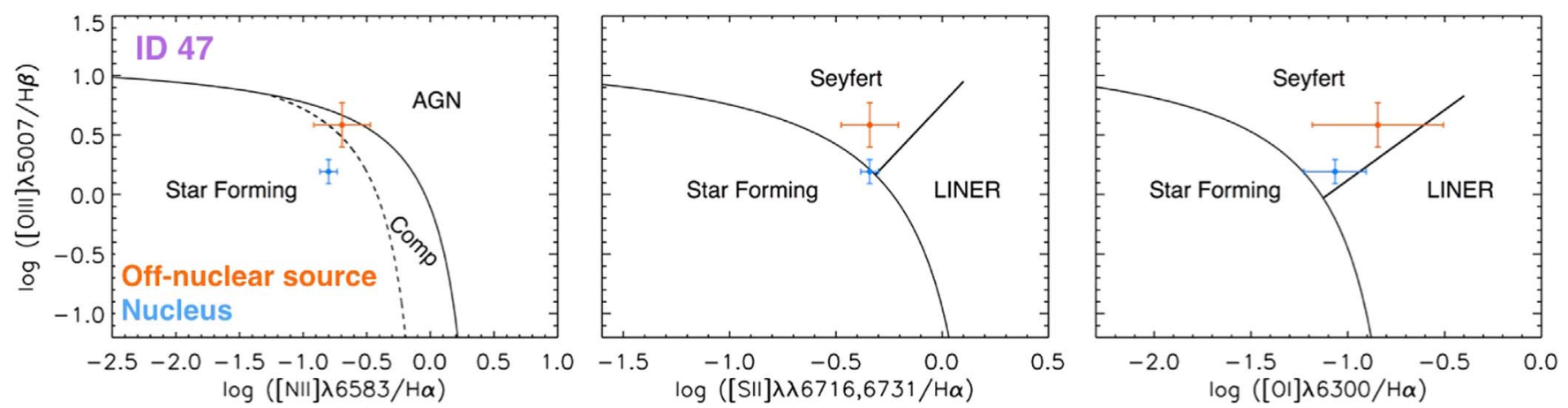

Figure 15. Optical emission-line diagnostic diagrams showing the nucleus and off-nuclear source in galaxy ID 47.
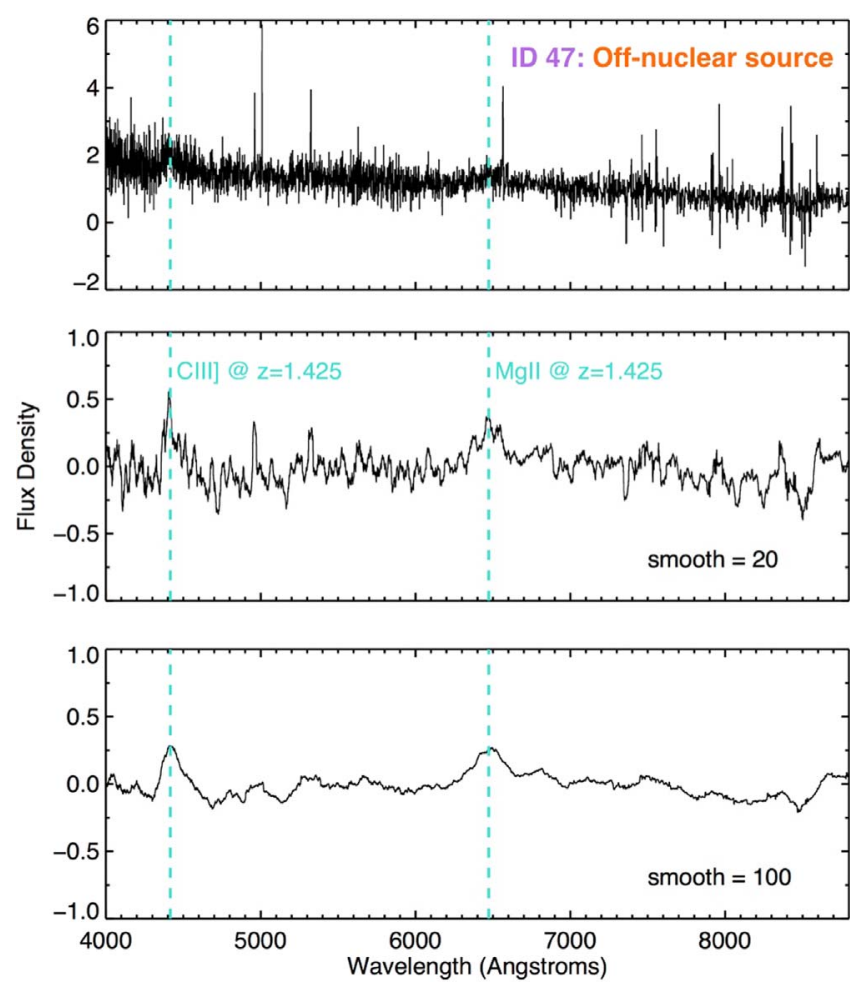

Figure 16. Top: SDSS spectrum of the off-nuclear radio source in ID 47 (Figure 3$)$ at the rest wavelength of the galaxy $(z=0.048)$. Middle: the same spectrum after subtracting the continuum and strong emission lines, smoothed with a boxcar average of 20 pixels. Bottom: same as middle panel, but smoothed with a boxcar average of 100 pixels. The turquoise dashed lines indicate redshifted broad UV lines commonly associated with quasars.

the narrow emission lines at the position of the offset source is associated with the dwarf galaxy. Moreover, our emission line measurements from the optical spectrum suggest the presence of an accreting massive black hole. The source falls in the Seyfert regions of the $[\mathrm{O} \mathrm{III}] / \mathrm{H} \beta$ versus $[\mathrm{S} \mathrm{II}] / \mathrm{H} \alpha$ and $[\mathrm{O} \mathrm{III}] / \mathrm{H} \beta$ versus $[\mathrm{O} \mathrm{I}] / \mathrm{H} \alpha$ diagnostic diagrams, as well as the AGN/star-forming composite region of the $[\mathrm{O} \mathrm{III}] / \mathrm{H} \beta$ versus $[\mathrm{N} \mathrm{II}] / \mathrm{H} \alpha$ BPT diagram (Figure 15).

While these findings suggest, at first glance, the presence of an offset massive BH in the dwarf galaxy, a closer inspection of the optical spectrum reveals broad, low-level emission lines that are consistent with $\mathrm{C}$ III] $\lambda 1909$ and $\operatorname{MgII} \lambda 2798$ at a redshift of $z=1.425$ (see Figure 16). This suggests the offset source is more likely a background quasar, which could account for the broad lines, the radio source, and the optical counterpart. The optical counterpart of the off-nuclear radio source is a variable point source in ground-based imaging. SDSS images of J1022-0055 were taken on 1999 March 22 and the DR15 catalog $g$ - and $r$-band magnitudes of the offnuclear source are $g=21.83 \pm 0.07 \mathrm{mag}$ and $r=21.22 \pm$ 0.06 mag. ${ }^{12}$ Images of J1022-0055 were also obtained as part of the Dark Energy Camera Legacy Survey (DECaLS) beginning in 2015 March, with the last $g$-band image taken in 2018 February and the last $r$-band image taken in 2017 March. The $g$ - and $r$-band magnitudes of the off-nuclear source in the DECaLS DR7 catalog (from the stacked images) are $g=22.98 \pm 0.02 \mathrm{mag}$ and $r=22.02 \pm 0.01 \mathrm{mag}$. Therefore, the off-nuclear source dimmed by $\sim 1$ mag between the SDSS and DECaLS imaging observations that were taken $\sim 16 \mathrm{yr}$ apart.

The narrow emission lines in the spectrum of the offset source can be attributed to gas in the dwarf galaxy that is ionized by an AGN, although the BH need not be at the location of the offset source. The nuclear spectrum of the galaxy shows hints of an AGN in the diagnostic diagrams shown in Figure 15. Therefore, it is plausible that there is indeed an AGN at the center of the dwarf galaxy, where the line emission due to accretion in the nuclear region is diluted by star formation but is more readily seen in the outskirts of the galaxy (e.g., Wylezalek et al. 2018). Nevertheless, it is somewhat surprising to find serendipitous optical evidence for an AGN in a dwarf galaxy that was selected because of a chance alignment with an unrelated radio source, potentially signaling an appreciable $\mathrm{BH}$ occupation fraction for galaxies of comparable mass $\left(M_{\star} \sim 3 \times 10^{9} M_{\odot} \sim M_{\star, \mathrm{LMC}}\right)$.

\section{ORCID iDs}

Amy E. Reines (1) https://orcid.org/0000-0001-7158-614X James J. Condon (1) https://orcid.org/0000-0003-4724-1939 Jeremy Darling (i) https://orcid.org/0000-0003-2511-2060

\section{References}

Anglés-Alcázar, D., Faucher-Giguère, C.-A., Quataert, E., et al. 2017, MNRAS, 472, L109

Aversa, A. G., Johnson, K. E., Brogan, C. L., Goss, W. M., \& Pisano, D. J. 2011, AJ, 141, 125

Baars, J. W. M., Genzel, R., Pauliny-Toth, I. I. K., \& Witzel, A. 1977, A\&A, 61,99

Baldassare, V. F., Geha, M., \& Greene, J. 2018, ApJ, 868, 152

Baldassare, V. F., Reines, A. E., Gallo, E., et al. 2016, ApJ, 829, 57

\footnotetext{
12 http://skyserver.sdss.org/dr15/en/tools/quicklook/summary.aspx? $\mathrm{ra}=10: 22: 27.86 \& \mathrm{dec}=-00: 55: 30.36$
} 
Baldassare, V. F., Reines, A. E., Gallo, E., \& Greene, J. E. 2015, ApJL, 809, L14

Baldassare, V. F., Reines, A. E., Gallo, E., \& Greene, J. E. 2017, ApJ, 836, 20 Baldwin, J. A., Phillips, M. M., \& Terlevich, R. 1981, PASP, 93, 5

Barth, A. J., Greene, J. E., \& Ho, L. C. 2008, AJ, 136, 1179

Becker, R. H., White, R. L., \& Helfand, D. J. 1995, ApJ, 450, 559

Bellovary, J. M., Cleary, C. E., Munshi, F., et al. 2019, MNRAS, 482, 2913

Bellovary, J. M., Governato, F., Quinn, T. R., et al. 2010, ApJL, 721, L148

Bentz, M. C., \& Manne-Nicholas, E. 2018, ApJ, 864, 146

Blanton, M. R., \& Roweis, S. 2007, AJ, 133, 734

Bruzual, G., \& Charlot, S. 2003, MNRAS, 344, 1000

Cann, J. M., Satyapal, S., Abel, N. P., et al. 2018, ApJ, 861, 142

Chen, C.-T. J., Brandt, W. N., Reines, A. E., et al. 2017, ApJ, 837, 48

Chomiuk, L., \& Wilcots, E. M. 2009, ApJ, 703, 370

Comerford, J. M., Pooley, D., Barrows, R. S., et al. 2015, ApJ, 806, 219

Condon, J. J. 1984, ApJ, 287, 461

Condon, J. J. 1992, ARA\&A, 30, 575

Condon, J. J., Matthews, A. M., \& Broderick, J. J. 2019, ApJ, 872, 148

Corbel, S., Dubus, G., Tomsick, J. A., et al. 2012, MNRAS, 421, 2947

Dong, X.-B., Ho, L. C., Yuan, W., et al. 2012, ApJ, 755, 167

Fu, H., Myers, A. D., Djorgovski, S. G., et al. 2015, ApJ, 799, 72

Gallo, E., Degenaar, N., \& van den Eijnden, J. 2018, MNRAS, 478, L132

Gallo, E., Treu, T., Jacob, J., et al. 2008, ApJ, 680, 154

Ghez, A. M., Salim, S., Weinberg, N. N., et al. 2008, ApJ, 689, 1044

Governato, F., Colpi, M., \& Maraschi, L. 1994, MNRAS, 271, 317

Greene, J. E., \& Ho, L. C. 2004, ApJ, 610, 722

Greene, J. E., \& Ho, L. C. 2007, ApJ, 670, 92

Groves, B. A., Heckman, T. M., \& Kauffmann, G. 2006, MNRAS, 371, 1559

Gwinn, C. R., Moran, J. M., \& Reid, M. J. 1992, ApJ, 393, 149

Habouzit, M., Volonteri, M., \& Dubois, Y. 2017, MNRAS, 468, 3935

Hainline, K. N., Reines, A. E., Greene, J. E., \& Stern, D. 2016, ApJ, 832, 119

Hao, C.-N., Kennicutt, R. C., Johnson, B. D., et al. 2011, ApJ, 741, 124

Ho, L. C. 2008, ARA\&A, 46, 475

Jarrett, T. H., Cohen, M., Masci, F., et al. 2011, ApJ, 735, 112

Jarrett, T. H., Masci, F., Tsai, C. W., et al. 2013, AJ, 145, 6

Johnson, K. E., Hunt, L. K., \& Reines, A. E. 2009, AJ, 137, 3788

Kauffmann, G., Heckman, T. M., Tremonti, C., et al. 2003, MNRAS, 346,1055

Kaviraj, S., Martin, G., \& Silk, J. 2019, MNRAS, 489, 12

Kennicutt, R. C., \& Evans, N. J. 2012, ARA\&A, 50, 531

Kennicutt, R. C., Jr. 1998, ARA\&A, 36, 189

Kepley, A. A., Reines, A. E., Johnson, K. E., \& Walker, L. M. 2014, AJ, 147,43

Kewley, L. J., Dopita, M. A., Sutherland, R. S., Heisler, C. A., \& Trevena, J. 2001, ApJ, 556, 121

Kewley, L. J., Groves, B., Kauffmann, G., \& Heckman, T. 2006, MNRAS, 372, 961

Kobulnicky, H. A., \& Johnson, K. E. 1999, ApJ, 527, 154

Kormendy, J., \& Ho, L. C. 2013, ARA\&A, 51, 511

Kormendy, J., \& Richstone, D. 1995, ARA\&A, 33, 581

Latif, M. A., \& Ferrara, A. 2016, PASA, 33, e051

Latimer, C. J., Reines, A. E., Plotkin, R. M., et al. 2019, ApJ, 884, 78

Lemons, S. M., Reines, A. E., Plotkin, R. M., Gallo, E., \& Greene, J. E. 2015, ApJ, 805, 12

Licquia, T. C., \& Newman, J. A. 2015, ApJ, 806, 96

Marleau, F. R., Clancy, D., Habas, R., \& Bianconi, M. 2017, A\&A, 602, A28
McConnell, N. J., \& Ma, C.-P. 2013, ApJ, 764, 184

Merritt, D., Milosavljević, M., Favata, M., Hughes, S. A., \& Holz, D. E. 2004, ApJL, 607, L9

Meurer, G. R., Heckman, T. M., Lehnert, M. D., Leitherer, C., \& Lowenthal, J. 1997, AJ, 114, 54

Mezcua, M., Civano, F., Marchesi, S., et al. 2018, MNRAS, 478, 2576

Mezcua, M., Suh, H., \& Civano, F. 2019, MNRAS, 488, 685

Mezger, P. G., Schraml, J., \& Terzian, Y. 1967, ApJ, 150, 807

Miller, B. P., Gallo, E., Greene, J. E., et al. 2015, ApJ, 799, 98

Moran, E. C., Filippenko, A. V., \& Chornock, R. 2002, ApJL, 579, L71

Moran, E. C., Shahinyan, K., Sugarman, H. R., Vélez, D. O., \& Eracleous, M. 2014, AJ, 148, 136

Mortlock, D. J., Warren, S. J., Venemans, B. P., et al. 2011, Natur, 474, 616 Müller-Sánchez, F., Comerford, J. M., Nevin, R., et al. 2015, ApJ, 813, 103 Natarajan, P. 2014, GReGr, 46, 1702

Pardo, K., Goulding, A. D., Greene, J. E., et al. 2016, ApJ, 831, 203

Plotkin, R. M., \& Reines, A. E. 2018, arXiv:1810.06814

Reed, J. E., Hester, J. J., Fabian, A. C., \& Winkler, P. F. 1995, ApJ, 440, 706

Reines, A. E., \& Comastri, A. 2016, PASA, 33, e054

Reines, A. E., \& Deller, A. T. 2012, ApJL, 750, L24

Reines, A. E., Greene, J. E., \& Geha, M. 2013, ApJ, 775, 116

Reines, A. E., Johnson, K. E., \& Goss, W. M. 2008, AJ, 135, 2222

Reines, A. E., Plotkin, R. M., Russell, T. D., et al. 2014, ApJL, 787, L30

Reines, A. E., Reynolds, M. T., Miller, J. M., et al. 2016, ApJL, 830, L35

Reines, A. E., Sivakoff, G. R., Johnson, K. E., \& Brogan, C. L. 2011, Natur, 470, 66

Reines, A. E., \& Volonteri, M. 2015, ApJ, 813, 82

Ricarte, A., \& Natarajan, P. 2018, MNRAS, 481, 3278

Sartori, L. F., Schawinski, K., Treister, E., et al. 2015, MNRAS, 454, 3722

Satyapal, S., Abel, N. P., \& Secrest, N. J. 2018, ApJ, 858, 38

Satyapal, S., Secrest, N. J., McAlpine, W., et al. 2014, ApJ, 784, 113

Schleicher, D. R. G. 2018, arXiv:1807.06055

Schramm, M., Silverman, J. D., Greene, J. E., et al. 2013, ApJ, 773, 150

Schulze, A., \& Wisotzki, L. 2010, A\&A, 516, A87

Schutte, Z., Reines, A., \& Greene, J. 2019, arXiv:1908.00020

Secrest, N. J., Satyapal, S., Gliozzi, M., et al. 2015, ApJ, 798, 38

Shimasaku, K., Fukugita, M., Doi, M., et al. 2001, AJ, 122, 1238

Stanimirović, S., Staveley-Smith, L., \& Jones, P. A. 2004, ApJ, 604, 176

Stern, D., Assef, R. J., Benford, D. J., et al. 2012, ApJ, 753, 30

Tremmel, M., Governato, F., Volonteri, M., \& Quinn, T. R. 2015, MNRAS, 451,1868

Turner, J. L., Beck, S. C., \& Ho, P. T. P. 2000, ApJL, 532, L109

Ulvestad, J. S. 2009, AJ, 138, 1529

Vacca, W. D., Garmany, C. D., \& Shull, J. M. 1996, ApJ, 460, 914

van Wassenhove, S., Volonteri, M., Walker, M. G., \& Gair, J. R. 2010, MNRAS, 408, 1139

Varenius, E., Conway, J. E., Batejat, F., et al. 2019, A\&A, 623, A173

Varenius, E., Conway, J. E., Martí-Vidal, I., et al. 2016, A\&A, 593, A86

Vito, F., Brandt, W. N., Yang, G., et al. 2018, MNRAS, 473, 2378

Volonteri, M. 2010, A\&ARv, 18, 279

Volonteri, M., Lodato, G., \& Natarajan, P. 2008, MNRAS, 383, 1079

Volonteri, M., \& Natarajan, P. 2009, MNRAS, 400, 1911

Volonteri, M., \& Perna, R. 2005, MNRAS, 358, 913

Volonteri, M., \& Reines, A. E. 2016, ApJL, 820, L6

Wu, X.-B., Wang, F., Fan, X., et al. 2015, Natur, 518, 512

Wylezalek, D., Zakamska, N. L., Greene, J. E., et al. 2018, MNRAS, 474, 1499 